\title{
Outlets of 2D invasion percolation and multiple-armed incipient infinite clusters
}

\author{
Michael Damron • Artëm Sapozhnikov
}

Received: 14 November 2009 / Revised: 25 January 2010 / Published online: 11 March 2010

(C) The Author(s) 2010. This article is published with open access at Springerlink.com

\begin{abstract}
We study invasion percolation in two dimensions, focusing on properties of the outlets of the invasion and their relation to critical percolation and to incipient infinite clusters (IICs). First we compute the exact decay rate of the distribution of both the weight of the $k$ th outlet and the volume of the $k$ th pond. Next we prove bounds for all moments of the distribution of the number of outlets in an annulus. This result leads to almost sure bounds for the number of outlets in a box $B\left(2^{n}\right)$ and for the decay rate of the weight of the $k$ th outlet to $p_{c}$. We then prove existence of multiple-armed IIC measures for any number of arms and for any color sequence which is alternating or monochromatic. We use these measures to study the invaded region near outlets and near edges in the invasion backbone far from the origin.
\end{abstract}

Keywords Invasion percolation - Invasion ponds - Critical percolation - Near critical percolation $\cdot$ Correlation length $\cdot$ Scaling relations $\cdot$ Incipient infinite cluster

Mathematics Subject Classification (2000) Primary 60K35 · $82 \mathrm{~B} 43$

Research funded by NSF grant OISE-0730136 and an NSF postdoctoral fellowship.

Research partially supported by the Netherlands Organisation for Scientific Research (NWO) under grant number 613.000.429.

M. Damron $(\varangle)$

Mathematics Department, Princeton University, Fine Hall,

Washington Rd., Princeton, NJ 08544, USA

e-mail:mdamron@math.princeton.edu

A. Sapozhnikov

EURANDOM, P.O. Box 513, 5600 MB Eindhoven, The Netherlands

e-mail: sapozhnikov@eurandom.tue.nl 


\section{Introduction}

\subsection{The model}

Invasion percolation is a stochastic growth model both introduced and numerically studied independently by $[1,14]$. Let $G=(V, E)$ be an infinite connected graph in which a distinguished vertex, the origin, is chosen. Let $\left(\tau_{e}\right)_{e \in E}$ be independent random variables, uniformly distributed on $[0,1]$. The invasion percolation cluster (IPC) of the origin on $G$ is defined as the limit of an increasing sequence $\left(G_{n}\right)$ of connected subgraphs of $G$ as follows. For an arbitrary subgraph $G^{\prime}=\left(V^{\prime}, E^{\prime}\right)$ of $G$, we define the outer edge boundary of $G^{\prime}$ as

$$
\Delta G^{\prime}=\left\{e=\langle x, y\rangle \in E: e \notin E^{\prime} \text {, but } x \in V^{\prime} \text { or } y \in V^{\prime}\right\} .
$$

We define $G_{0}$ to be the origin. Once the graph $G_{i}=\left(V_{i}, E_{i}\right)$ is defined, we select the edge $e_{i+1}$ that minimizes $\tau$ on $\Delta G_{i}$. We take $E_{i+1}=E_{i} \cup\left\{e_{i+1}\right\}$ and let $G_{i+1}$ be the graph induced by the edge set $E_{i+1}$. The graph $G_{i}$ is called the invaded region at time $i$. Let $E_{\infty}=\cup_{i=0}^{\infty} E_{i}$ and $V_{\infty}=\cup_{i=0}^{\infty} V_{i}$. Finally, define the IPC $\mathcal{S}=\left(V_{\infty}, E_{\infty}\right)$.

In this paper, we study invasion percolation on two-dimensional lattices; however, for simplicity we restrict ourselves hereafter to the square lattice $\mathbb{Z}^{2}$ and denote by $\mathbb{E}^{2}$ the set of nearest-neighbor edges. The results of this paper still hold for lattices which are invariant under reflection in one of the coordinate axes and under rotation around the origin by some angle. In particular, this includes the triangular and honeycomb lattices.

We define Bernoulli percolation using the random variables $\tau_{e}$ to make a coupling with the invasion immediate. For any $p \in[0,1]$ we say that an edge $e \in \mathbb{E}^{2}$ is $p$-open if $\tau_{e}<p$ and $p$-closed otherwise. It is obvious that the resulting random graph of $p$-open edges has the same distribution as the one obtained by declaring each edge of $\mathbb{E}^{2}$ open with probability $p$ and closed with probability $1-p$, independently of the state of all other edges. The percolation probability $\theta(p)$ is the probability that the origin is in the infinite cluster of $p$-open edges. There is a critical probability $p_{c}=\inf \{p: \theta(p)>0\} \in(0,1)$. For general background on Bernoulli percolation we refer the reader to [9].

In [3], it was shown that, for any $p>p_{c}$, the invasion on $\left(\mathbb{Z}^{d}, \mathbb{E}^{d}\right)$ intersects the infinite $p$-open cluster with probability one. The definition of the invasion mechanism implies that if the invasion reaches the $p$-open infinite cluster for some $p$, it will never leave this cluster. Combining these two facts yields that if $e_{i}$ is the edge added at step $i$ then $\lim \sup _{i \rightarrow \infty} \tau_{e_{i}}=p_{c}$. It is well-known that for Bernoulli percolation on $\left(\mathbb{Z}^{2}, \mathbb{E}^{2}\right)$, the percolation probability at $p_{c}$ is 0 . This implies that, for infinitely many values of $i$, the weight $\tau_{e_{i}}>p_{c}$. The last two results give that $\hat{\tau}_{1}=\max \left\{\tau_{e}: e \in E_{\infty}\right\}$ exists and is greater than $p_{c}$. The above maximum is attained at an edge which we shall call $\hat{e}_{1}$. Suppose that $\hat{e}_{1}$ is invaded at step $i_{1}$, i.e. $\hat{e}_{1}=e_{i_{1}}$. Following the terminology of [16], we call the graph $G_{i_{1}-1}$ the first pond of the invasion, denoting it by the symbol $\hat{V}_{1}$, and we call the edge $\hat{e}_{1}$ the first outlet. The second pond of the invasion is defined similarly. Note that a simple extension of the above argument implies that $\hat{\tau}_{2}=\max \left\{\tau_{e_{i}}: e_{i} \in E_{\infty}, i>i_{1}\right\}$ exists and is greater than $p_{c}$. If we assume that $\hat{\tau}_{2}$ 
is taken on the edge $\hat{e}_{2}$ at step $i_{2}$, we call the graph $G_{i_{2}-1} \backslash G_{i_{1}-1}$ the second pond of the invasion, and we denote it $\hat{V}_{2}$. The edge $\hat{e}_{2}$ is called the second outlet. The further ponds $\hat{V}_{k}$ and outlets $\hat{e}_{k}$ are defined analogously. For a hydrological interpretation of the ponds we refer the reader to [20].

In this paper we study the sequence of outlets $\left(\hat{e}_{k}\right)$ and the sequence of their weights $\left(\hat{\tau}_{k}\right)$. In Theorem 1.1 we give the asymptotic behaviour for the distribution of $\hat{\tau}_{k}$ for any fixed $k$. For $k>1$, we compute the exact decay rate of the distribution of the size of the $k$ th pond in Theorem 1.2. This result can be also seen as a statement about the sequence of steps $i_{k}$ at which $\hat{e}_{k}$ are invaded. In Theorem 1.3, we find uniform bounds on all moments of the number of outlets in an annulus. We use this result in Theorem 1.4 to derive almost sure bounds on the number of outlets in a box $B\left(2^{n}\right)$. An important consequence of Theorem 1.4 is Corollary 1.1; it states almost sure bounds on the difference $\left(\hat{\tau}_{k}-p_{c}\right)$ and on the radii of the ponds.

In Theorem 1.6 we prove the existence of an IIC with several infinite $p_{c}$-open and $p_{c}$-closed paths from a neighborhood of the origin. Last, we show in Theorems 1.8 and 1.9 that the local description of the invaded region near the backbone of the IPC far away from the origin is given by the IIC with two infinite $p_{c}$-open paths, and the local description of the invaded region near an outlet of the IPC far away from the origin is given by the IIC with two infinite $p_{c}$-open paths and two infinite $p_{c}$-closed paths so that these paths alternate.

\subsection{Notation}

In this section we collect most of the notation and the definitions used in the paper.

For $a \in \mathbb{R}$, we write $|a|$ for the absolute value of $a$, and, for a site $x=\left(x_{1}, x_{2}\right) \in \mathbb{Z}^{2}$, we write $|x|$ for $\max \left(\left|x_{1}\right|,\left|x_{2}\right|\right)$. For $n>0$ and $x \in \mathbb{Z}^{2}$, let $B(x, n)=\left\{y \in \mathbb{Z}^{2}\right.$ : $|y-x| \leq n\}$ and $\partial B(x, n)=\left\{y \in \mathbb{Z}^{2}:|y-x|=n\right\}$. We write $B(n)$ for $B(0, n)$ and $\partial B(n)$ for $\partial B(0, n)$. For $m<n$ and $x \in \mathbb{Z}^{2}$, we define the annulus $A n n(x ; m, n)=$ $B(x, n) \backslash B(x, m)$. We write $A n n(m, n)$ for $A n n(0 ; m, n)$.

We consider the square lattice $\left(\mathbb{Z}^{2}, \mathbb{E}^{2}\right)$, where $\mathbb{E}^{2}=\left\{\langle x, y\rangle \in \mathbb{Z}^{2} \times \mathbb{Z}^{2}:|x-y|=\right.$ $1\}$. Let $\left(\mathbb{Z}^{2}\right)^{*}=(1 / 2,1 / 2)+\mathbb{Z}^{2}$ and $\left(\mathbb{E}^{2}\right)^{*}=(1 / 2,1 / 2)+\mathbb{E}^{2}$ be the vertices and the edges of the dual lattice. For $x \in \mathbb{Z}^{2}$, we write $x^{*}$ for $x+(1 / 2,1 / 2)$. For an edge $e \in \mathbb{E}^{2}$ we denote its endpoints (left respectively right or bottom respectively top) by $e_{x}, e_{y} \in \mathbb{Z}^{2}$. The edge $e^{*}=\left\langle e_{x}+(1 / 2,1 / 2), e_{y}-(1 / 2,1 / 2)\right\rangle$ is called the dual edge to $e$. Its endpoints (bottom respectively top or left respectively right) are denoted by $e_{x}^{*}$ and $e_{y}^{*}$. Note that, in general, $e_{x}^{*}$ and $e_{y}^{*}$ are not the same as $\left(e_{x}\right)^{*}$ and $\left(e_{y}\right)^{*}$. For a subset $\mathcal{K} \subset \mathbb{Z}^{2}$, let $\mathcal{K}^{*}=(1 / 2,1 / 2)+\mathcal{K}$. We say that an edge $e \in \mathbb{E}^{2}$ is in $\mathcal{K} \subset \mathbb{Z}^{2}$ if both its endpoints are in $\mathcal{K}$. For any graph $\mathcal{G}$ we write $|\mathcal{G}|$ for the number of vertices in $\mathcal{G}$.

Let $\left(\tau_{e}\right)_{e \in \mathbb{E}^{2}}$ be independent random variables, uniformly distributed on $[0,1]$, indexed by edges. We call $\tau_{e}$ the weight of an edge $e$. We define the weight of an edge $e^{*}$ as $\tau_{e^{*}}=\tau_{e}$. We denote the underlying probability measure by $\mathbb{P}$ and the space of configurations by $\left([0,1]^{\mathbb{E}^{2}}, \mathcal{F}\right)$, where $\mathcal{F}$ is the natural $\sigma$-field on $[0,1]^{\mathbb{E}^{2}}$. We say that an edge $e$ is $p$-open if $\tau_{e}<p$ and $p$-closed if $\tau_{e} \geq p$. An edge $e^{*}$ is $p$-open if $e$ is $p$-open, and it is $p$-closed if $e$ is $p$-closed. Accordingly, for $p \in[0,1]$, we define 
the edge configuration $\omega_{p} \in\{0,1\}^{\mathbb{Z}^{2}}$ by $\omega_{p}(e)=1$ if $\tau_{e}<p$ and 0 if $\tau_{e} \geq p$. We make a similar definition for $\omega_{p}^{*}$, the dual edge configuration. The event that two sets of sites $\mathcal{K}_{1}, \mathcal{K}_{2} \subset \mathbb{Z}^{2}$ are connected by a $p$-open path is denoted by $\mathcal{K}_{1} \stackrel{p}{\longleftrightarrow} \mathcal{K}_{2}$, and the event that two sets of sites $\mathcal{K}_{1}^{*}, \mathcal{K}_{2}^{*} \subset\left(\mathbb{Z}^{2}\right)^{*}$ are connected by a $p$-closed path in the dual lattice is denoted by $\mathcal{K}_{1}^{*} \stackrel{p^{*}}{\longleftrightarrow} \mathcal{K}_{2}^{*}$. For any $n \geq 1$ and $p \in[0,1]$, we define the event

$$
\begin{aligned}
B_{n, p}= & \{\text { There is a } p \text {-closed circuit with radius at least } n \\
& \text { around the origin in the dual lattice }\}
\end{aligned}
$$

For $p \in[0,1]$, we consider a probability space $\left(\Omega_{p}, \mathcal{F}_{p}, \mathbb{P}_{p}\right)$, where $\Omega_{p}=\{0,1\} \mathbb{E}^{\mathbb{E}^{2}}$, $\mathcal{F}_{p}$ is the $\sigma$-field generated by the finite-dimensional cylinders of $\Omega_{p}$, and $\mathbb{P}_{p}$ is a product measure on $\left(\Omega_{p}, \mathcal{F}_{p}\right)$, defined as $\mathbb{P}_{p}=\prod_{e \in \mathbb{E}^{2}} \mu_{e}$, where $\mu_{e}$ is the probability measure on $\{0,1\}$ with $\mathbb{P}_{p}\left(\omega_{e}=1\right)=\mu_{e}(\{1\})=1-\mu_{e}(\{0\})=1-\mathbb{P}_{p}\left(\omega_{e}=0\right)=p$. We say that an edge $e$ is open or occupied if $\omega_{e}=1$, and $e$ is closed or vacant if $\omega_{e}=0$. We say that an edge $e^{*}$ is open or occupied if $e$ is open, and it is closed or vacant if $e$ is closed. The event that two sets of sites $\mathcal{K}_{1}, \mathcal{K}_{2} \subset \mathbb{Z}^{2}$ are connected by an open path is denoted by $\mathcal{K}_{1} \leftrightarrow \mathcal{K}_{2}$, and the event that two sets of sites $\mathcal{K}_{1}^{*}, \mathcal{K}_{2}^{*} \subset \mathbb{Z}^{2}$ are connected by a closed path in the dual lattice is denoted by $\mathcal{K}_{1}^{*} \stackrel{*}{\leftrightarrow} \mathcal{K}_{2}^{*}$. For any $n \geq 1$ and $p \in[0,1]$, let $\pi_{n}=\mathbb{P}_{p_{c}}(0 \leftrightarrow \partial B(n))$ and $\pi(n, p)=\mathbb{P}_{p}(0 \leftrightarrow \partial B(n))$. Also define the event

$$
\begin{gathered}
B_{n}=\{\text { There is a closed circuit with radius at least } n \text { around the origin in the } \\
\text { dual lattice }\}
\end{gathered}
$$

For any $k \geq 1$, let $\hat{R}_{k}$ be the radius of the union of the first $k$ ponds. In other words, $\hat{R}_{k}=\max \left\{|x|: x \in \cup_{j=1}^{k} \hat{V}_{j}\right\}$. For two functions $g$ and $h$ from a set $\mathcal{X}$ to $\mathbb{R}$, we write $g(z) \asymp h(z)$ to indicate that $g(z) / h(z)$ is bounded away from 0 and $\infty$, uniformly in $z \in \mathcal{X}$. Throughout this paper we write $\log$ for $\log _{2}$. We also write $\mathbb{P}_{c r}$ for $\mathbb{P}_{p_{c}}$. All the constants $\left(C_{i}\right)$ in the proofs are strictly positive and finite. Their exact values may be different from proof to proof.

\subsection{Main results}

\subsubsection{Weight of the kth outlet}

Let $\hat{\tau}_{k}$ be the weight of the $k$ th outlet, as defined in Sect. 1.1.

Theorem 1.1 For any $k \geq 1$,

$$
\mathbb{P}\left(\hat{\tau}_{k}<p\right) \asymp(\log L(p))^{k-1} \theta(p), \quad p>p_{c},
$$

where the correlation length $L(p)$ is defined in Sect. 2. 
Remark 1 Note that the statement is trivial in the case $k=1$. Indeed, it follows from the definition of the invasion that $\mathbb{P}\left(\hat{\tau}_{1}<p\right)=\theta(p)$ for all $p$.

\subsubsection{Volumes of the ponds}

Theorem 1.2 For any $k \geq 1$,

$$
\mathbb{P}\left(\left|\hat{V}_{k}\right| \geq n^{2} \pi_{n}\right) \asymp(\log n)^{k-1} \pi_{n}, \quad n \geq 2 .
$$

In particular,

$$
\mathbb{P}\left(\left|\hat{V}_{k}\right| \geq n\right) \asymp(\log n)^{k-1} \mathbb{P}_{c r}(|C(0)| \geq n), \quad n \geq 2 .
$$

Remark 2 The case $k=1$ is considered in [20].

Remark 3 The second set of inequalities follows from the first one using the relations $\mathbb{P}_{c r}\left(|C(0)| \geq n^{2} \pi_{n}\right) \asymp \pi_{n}$ (see [20, Theorem 2]) and $\log \left(n^{2} \pi_{n}\right) \asymp \log n$.

Remark 4 Let $i_{k}$ be the index such that $e_{i_{k}}=\hat{e}_{k}$. Then $i_{k}$ is comparable to $\left|\hat{V}_{1}\right|+\cdots+$ $\left|\hat{V}_{k}\right|$. Therefore the statements (1.2) and (1.3) hold with $\left|\hat{V}_{k}\right|$ replaced by $i_{k}$.

\subsubsection{Almost sure bounds}

For any $m<n$, let $O(m, n)$ be the number of outlets in $A n n(m, n)$, and let $O(n)$ be the number of outlets in $B(n)$. We first give $n$-independent bounds on all moments of $O(n, 2 n)$.

Theorem 1.3 There exists $c_{1}>0$ such that for all $t, n \geq 1$,

$$
\mathbb{E}\left(O(n, 2 n)^{t}\right) \leq\left(c_{1} t\right)^{3 t}
$$

In particular, there exists $c_{2}, \lambda>0$ such that for all $n$,

$$
\mathbb{E}\left(\exp \left(\lambda O(n, 2 n)^{1 / 3}\right)\right)<c_{2}
$$

Next we show almost sure bounds on the sequence of random variables $\left(O\left(2^{n}\right)\right)_{n \geq 1}$.

Theorem 1.4 There exists $c_{3}, c_{4}>0$ such that with probability one, for all large $n$,

$$
c_{3} n \leq O\left(2^{n}\right) \leq c_{4} n
$$

Theorem 1.4 implies related bounds on the convergence rate of the weights $\hat{\tau}_{k}$ to $p_{c}$ and on the growth of the radii $\left(\hat{R}_{k}\right)_{k \geq 1}$.

Corollary 1.1 1. There exists $c_{5}$ and $c_{6}$ with $1<c_{5}, c_{6}<\infty$ such that with probability one, for all large $k$,

$$
\left(c_{5}\right)^{k} \leq \hat{R}_{k} \leq\left(c_{6}\right)^{k}
$$


2. There exists $c_{7}$ and $c_{8}$ with $0<c_{7}, c_{8}<1$ such that with probability one, for all large $k$,

$$
\left(c_{7}\right)^{k} \leq \hat{\tau}_{k}-p_{c} \leq\left(c_{8}\right)^{k}
$$

Remark 5 Asymptotics of various ponds statistics as well as CLT-type and large deviations results for deviations of those quantities away from their limits are studied in [8] for invasion percolation on regular trees. Not only do the results in [8] imply exponential almost sure bounds similar to (1.7) and (1.8), they are very explicit. For instance, it is shown that $\lim _{k \rightarrow \infty} \frac{1}{k} \ln \left(\hat{\tau}_{k}-p_{c}\right)=-1$ and $\lim _{k \rightarrow \infty} \frac{1}{k} \ln \left(\hat{R}_{k}\right)=1$ a.s.

Our last theorem concerns ratios of successive terms of the sequence $\left(\hat{\tau}_{k}-p_{c}\right)_{k \geq 1}$.

Theorem 1.5 With probability one, the set

$$
\left\{\frac{\hat{\tau}_{k+1}-p_{c}}{\hat{\tau}_{k}-p_{c}}: k \geq 1\right\}
$$

is a dense subset of $[0,1]$.

\subsubsection{Outlets and multiple-armed IICs}

First we recall the definition of the incipient infinite cluster from [12]. It is shown in [12] that the limit

$$
v(E)=\lim _{N \rightarrow \infty} \mathbb{P}_{c r}(E \mid 0 \leftrightarrow \partial B(N))
$$

exists for any event $E$ that depends on the state of finitely many edges in $\mathbb{E}^{2}$. The unique extension of $v$ to a probability measure on configurations of open and closed edges exists. Under this measure, the open cluster of the origin is a.s. infinite. It is called the incipient infinite cluster (IIC). In Theorem 1.7 [10, Theorem 3], a relation between IPC and IIC is given.

In this section we introduce multiple-armed IIC measures (Theorem 1.6) and study their relation to invasion percolation (Theorems $1.8,1.9$ ). For this, let $k \geq 1$ and $\sigma \in$ \{open, closed ${ }^{k}$. Let $r_{1}$ be the number of 'open' entries in $\sigma$ and let $r_{2}$ be the number of 'closed' entries in $\sigma$. For $l<n$ such that $|\partial B(l)|>|\sigma|$, we say that $B(l)$ is $\sigma$-connected to $\partial B(n)$, denoted $B(l) \leftrightarrow_{\sigma} \partial B(n)$, if there exist $r_{1}$ disjoint open paths between $B(l)$ and $\partial B(n)$ and $r_{2}$ disjoint dual closed paths between $B(l)^{*}$ and $\partial B(n)^{*}$ such that the relative counterclockwise arrangement of these paths is given by $\sigma$. In the definition above we allow $n=\infty$, in this case we write $B(l) \leftrightarrow_{\sigma} \infty$.

Theorem 1.6 Suppose that $\sigma$ is alternating and let $l$ be the minimal number such that $|\partial B(l)| \geq|\sigma|$. For every cylinder event $E$, the limit

$$
v_{\sigma}(E)=\lim _{n \rightarrow \infty} \mathbb{P}_{c r}\left(E \mid B(l) \leftrightarrow_{\sigma} \partial B(n)\right)
$$


exists. For the unique extension of $v_{\sigma}$ to a probability measure on the configurations of open and closed edges,

$$
v_{\sigma}\left(B(l) \leftrightarrow_{\sigma} \infty\right)=1
$$

We call the resulting measure $v_{\sigma}$ the $\sigma$-incipient infinite cluster measure.

Remark 6 Note that Kesten's IIC measure corresponds to the case $\sigma=\{$ open $\}$ with $l=0$. One can check that Kesten's original proof [12] also works for the case $\sigma=$ \{open, open\}. We use this second IIC measure in Theorem 1.8.

Remark 7 The proof we present of Theorem 1.6 can be easily modified to give the existence of IICs for $\sigma$ 's which either do not contain neighboring open paths or do not contain neighboring closed paths (here we take the first and last elements of $\sigma$ to be neighbors). In particular, it works for any 3-arm IIC and for monochromatic IICs. In the case when there are neighboring open paths (but no neighboring closed paths) one needs to change the proof by considering closed circuits with defects instead of open circuits.

Define $\mathcal{O}=\left\{\hat{e}_{k}: k \geq 1\right\}$, the set of outlets of the invasion and let $\mathcal{B}$ be the backbone, i.e., those vertices which are connected in the IPC of the origin by two disjoint paths, one to the origin and one to $\infty$. For any vertex $v$, define the shift operator $\theta_{v}$ on configurations $\omega$ so that for any edge $e, \theta_{v}(\omega)(e)=\omega(e-v)$, where $e-v=\left\langle e_{x}-v, e_{y}-v\right\rangle$. For any event $E$, define

$$
\theta_{v} E=\left\{\theta_{v}(\omega): \omega \in E\right\}
$$

and if $\mathcal{K}$ is a set of edges in $\mathbb{E}^{2}$, define

$$
E_{\mathcal{K}}=\{\mathcal{K} \subset \mathcal{S}\}, \quad \theta_{v} \mathcal{K}=\left\{e \in \mathbb{E}^{2}: e-v \in \mathcal{K}\right\}, \quad \text { and } \quad \Theta_{v} E_{\mathcal{K}}=\left\{\theta_{v} \mathcal{K} \subset \mathcal{S}\right\}
$$

For an edge $e$, let $\rho_{e}$ be the rotation of the lattice around the origin that maps $e-e_{x}$ to $\langle(0,0),(1,0)\rangle$. We define the operator $\theta_{e}$ on configurations $\omega$ so that for any edge $f, \theta_{e}(\omega)(f)=\omega\left(\rho_{e}\left(f-e_{x}\right)\right)$. We define $\theta_{e} E, \theta_{e} \mathcal{K}$, and $\Theta_{e} E_{\mathcal{K}}$ similarly. Let $E_{\mathcal{K}}^{\prime}$ be the event that $\mathcal{K}$ is contained in the cluster of the origin. We recall [10, Theorem 3], which states that asymptotically the distribution of invaded edges near a vertex $v$ is given by the IIC measure.

Theorem 1.7 Let $E$ be an event which depends on finitely many values $\omega_{p_{c}}(\cdot)$ and let $\mathcal{K} \subset \mathbb{E}^{2}$ be finite.

$$
\lim _{|v| \rightarrow \infty} \mathbb{P}\left(\theta_{v} E \mid v \in \mathcal{S}\right)=v(E) \quad \text { and } \lim _{|v| \rightarrow \infty} \mathbb{P}\left(\Theta_{v} E_{\mathcal{K}} \mid v \in \mathcal{S}\right)=v\left(E_{\mathcal{K}}^{\prime}\right)
$$

where the measure on the right is the IIC measure.

We are interested in the distribution of invaded edges near the backbone (Theorem 1.8) or near an outlet (Theorem 1.9). While the analysis of the distribution of the 
invaded edges near the backbone is very similar to the proof of Theorem 1.7, the study of the distribution of the invaded edges near an outlet is more involved. Define $\tilde{v}^{2,0}$ to be the measure constructed in the same way as $v^{2,0}$ except that we condition that the origin is connected to $\partial B(n)$ by two disjoint open paths and take $n$ to $\infty$. Define $\tilde{v}^{2,2}$ similarly, but by conditioning that the endpoints of the edge $e_{0}=\langle(0,0),(1,0)\rangle$ are connected to $\partial B(n)$ by two disjoint open paths and that the endpoints of $e_{0}^{*}$ are connected to $\partial B(n)^{*}$ by two disjoint closed dual paths and taking $n$ to $\infty$. Obvious modifications of Theorem 1.6 hold for these measures.

Theorem 1.8 Let $E$ be an event which depends on finitely many values $\omega_{p_{c}}(\cdot)$ and let $\mathcal{K} \subset \mathbb{E}^{2}$ be finite.

$$
\lim _{|v| \rightarrow \infty} \mathbb{P}\left(\theta_{v} E \mid v \in \mathcal{B}\right)=\tilde{v}^{2,0}(E) \quad \text { and } \lim _{|v| \rightarrow \infty} \mathbb{P}\left(\Theta_{v} E_{\mathcal{K}} \mid v \in \mathcal{B}\right)=\tilde{v}^{2,0}\left(E_{\mathcal{K}}^{\prime}\right)
$$

Proof Similar to the proof of [10, Theorem 3].

Theorem 1.9 Let $E$ be an event which depends on finitely many values $\omega_{p_{c}}(\cdot)$ (but not on $\left.\omega_{p_{c}}\left(e_{0}\right)\right)$, and let $\mathcal{K}$ be a finite set of edges such that $e_{0} \notin \mathcal{K}$.

$$
\lim _{|e| \rightarrow \infty} \mathbb{P}\left(\theta_{e} E \mid e \in \mathcal{O}\right)=\tilde{v}^{2,2}(E) \quad \text { and } \quad \lim _{|e| \rightarrow \infty} \mathbb{P}\left(\Theta_{e} E_{\mathcal{K}} \mid e \in \mathcal{O}\right)=\tilde{v}^{2,2}\left(E_{\mathcal{K}}^{\prime}\right)
$$

\subsection{Structure of the paper}

We define the correlation length and state some of its properties in Sect. 2. We prove Theorems 1.1 and 1.2 in Sects. 3 and 4, respectively. The proofs of Theorems 1.3-1.5 are in Sect. 5: the proof of Theorem 1.3 is in Sect. 5.1; the proofs of Theorem 1.4 and Corollary 1.1 are in Sect. 5.2; and the proof of Theorem 1.5 is in Sect. 5.3. We prove Theorem 1.6 in Sect. 6 and Theorem 1.9 in Sect. 7. For the notation in Sects. 3-7 we refer the reader to Sect. 1.2.

\section{Correlation length and preliminary results}

In this section we define the correlation length that will play a crucial role in our proofs. The correlation length was introduced in [2] and further studied in [13].

\subsection{Correlation length}

For $m, n$ positive integers and $p \in\left(p_{c}, 1\right]$ let

$$
\sigma(n, m, p)=\mathbb{P}_{p}(\text { there is an open horizontal crossing of }[0, n] \times[0, m])
$$

Given $\varepsilon>0$ and $p>p_{c}$, we define

$$
L(p, \varepsilon)=\min \{n: \sigma(n, n, p) \geq 1-\varepsilon\} .
$$


$L(p, \varepsilon)$ is called the finite-size scaling correlation length and it is known that $L(p, \varepsilon)$ scales like the usual correlation length (see [13]). It was also shown in [13] that the scaling of $L(p, \varepsilon)$ is independent of $\varepsilon$ given that it is small enough, i.e. there exists $\varepsilon_{0}>0$ such that for all $0<\varepsilon_{1}, \varepsilon_{2} \leq \varepsilon_{0}$ we have $L\left(p, \varepsilon_{1}\right) \asymp L\left(p, \varepsilon_{2}\right)$. For simplicity we will write $L(p)=L\left(p, \varepsilon_{0}\right)$ for the entire paper. We also define

$$
p_{n}=\sup \{p: L(p)>n\} .
$$

It is easy to see that $L(p) \rightarrow \infty$ as $p \rightarrow p_{c}$ and $L(p) \rightarrow 0$ as $p \rightarrow 1$. In particular, the probability $p_{n}$ is well-defined. It is clear from the definitions of $L(p)$ and $p_{n}$ and from the RSW theorem that, for positive integers $k$ and $l$, there exists $\delta_{k, l}>0$ such that, for any positive integer $n$ and for all $p \in\left[p_{c}, p_{n}\right]$,

$$
\mathbb{P}_{p}(\text { there is an open horizontal crossing of }[0, k n] \times[0, \ln ])>\delta_{k, l}
$$

and

$\mathbb{P}_{p}$ (there is a closed horizontal dual crossing of $\left.([0, k n] \times[0, \ln ])^{*}\right)>\delta_{k, l}$.

By the FKG inequality and a standard gluing argument [9, Section 11.7] we get that, for positive integers $n$ and $k \geq 2$ and for all $p \in\left[p_{c}, p_{n}\right]$,

$$
\mathbb{P}_{p}(A n n(n, k n) \text { contains an open circuit around the origin })>\left(\delta_{k, k-2}\right)^{4}
$$

and

$$
\mathbb{P}_{p}\left(\operatorname{Ann}(n, k n)^{*} \text { contains a closed dual circuit around the origin }\right)>\left(\delta_{2 k, k-1}\right)^{4} .
$$

\subsection{Preliminary results}

For any positive $l$ we define $\log ^{(0)} l=l$ and $\log ^{(j)} l=\log (\log (j-1) l)$ for all $j \geq 1$, as long as the right-hand side is well defined. For $l>10$, let

$$
\log ^{*} l=\min \left\{j>0: \log ^{(j)} l \text { is well-defined and } \log ^{(j)} l \leq 10\right\} .
$$

Our choice of the constant 10 is quite arbitrary, we could take any other large enough positive number instead of 10 . For $l>10$, let

$$
p_{l}(j)= \begin{cases}\inf \left\{p>p_{c}: L(p) \leq \frac{l}{\left.C_{*} \log ^{(j)}\right\}}\right\} & \text { if } j \in\left(0, \log ^{*} l\right) \\ p_{c} & \text { if } j \geq \log ^{*} l \\ 1 & \text { if } j=0\end{cases}
$$

The value of $C_{*}$ will be chosen differently in each proof. For any $C_{*}$, notice that there exists a universal constant $L_{0}\left(C_{*}\right)>10$ such that $p_{l}(j)$ are well-defined if $l>L_{0}\left(C_{*}\right)$ and non-increasing in $l$. The last observation follows from monotonicity of $L(p)$ and 
the fact that the functions $l / \log ^{(j)} l$ are non-decreasing in $l$ for $j \in\left(0, \log ^{*} l\right)$ and $l \geq 3$.

We give the following results without proofs.

1. ([10, (2.10)]) There exists a universal constant $D_{1}$ such that, for every $l>L_{0}\left(C_{*}\right)$ and $j \in\left(0, \log ^{*} l\right)$,

$$
C_{*} \log ^{(j)} l \leq \frac{l}{L\left(p_{l}(j)\right)} \leq D_{1} C_{*} \log ^{(j)} l .
$$

2. ([13, Theorem 2]) There is a constant $D_{2}$ such that, for all $p>p_{c}$,

$$
\theta(p) \leq \mathbb{P}_{p}[0 \leftrightarrow \partial B(L(p))] \leq D_{2} \mathbb{P}_{c r}[0 \leftrightarrow \partial B(L(p))]
$$

where $\theta(p)=\mathbb{P}_{p}(0 \rightarrow \infty)$ is the percolation function for Bernoulli percolation.

3. ([17, Section 4]) There is a constant $D_{3}$ such that, for all $n \geq 1$,

$$
\mathbb{P}_{p_{n}}(B(n) \leftrightarrow \infty) \geq D_{3}
$$

4. ([13, (3.61)]) There is a constant $D_{4}$ such that, for all positive integers $r \leq s$,

$$
\frac{\mathbb{P}_{c r}(0 \leftrightarrow \partial B(s))}{\mathbb{P}_{c r}(0 \leftrightarrow \partial B(r))} \geq D_{4} \sqrt{\frac{r}{s}} .
$$

5. There exist positive constants $D_{5}$ and $D_{6}$ such that, for all $p>p_{c}$,

$$
\mathbb{P}_{p}\left(B_{n}\right) \leq D_{5} \exp \left\{-D_{6} \frac{n}{L(p)}\right\}
$$

It follows, for example, from [10, (2.6) and (2.8)] (see also [18, Lemma 37 and Remark 38]).

6. ([18, Proposition 34]) Fix $e=\langle(0,0),(1,0)\rangle$, and let $A_{n}^{2,2}$ be the event that $e_{x}$ and $e_{y}$ are connected to $\partial B(n)$ by open paths, and $e_{x}^{*}$ and $e_{y}^{*}$ are connected to $\partial B(n)^{*}$ by closed dual paths. Note that these four paths are disjoint and alternate. Then

$$
\left(p_{n}-p_{c}\right) n^{2} \mathbb{P}_{c r}\left(A_{n}^{2,2}\right) \asymp 1, \quad n \geq 1 .
$$

\section{Proof of Theorem 1.1}

We give the proof for the case $k=2$. The proof for $k \geq 3$ is similar to the proof for $k=2$, and we omit the details. Note that [13, Theorem 2] it is sufficient to prove that

$$
\mathbb{P}\left(\hat{\tau}_{2}<p\right) \asymp(\log L(p)) \mathbb{P}_{c r}(0 \leftrightarrow \partial B(L(p)))
$$


We first prove the upper bound. We partition the box $B(L(p))$ into $\lfloor\log L(p)\rfloor$ disjoint annuli:

$$
\begin{aligned}
\mathbb{P}\left(\hat{\tau}_{2}<p\right) \leq & \mathbb{P}\left(\hat{R}_{1} \geq L(p)\right) \\
& +\sum_{k=0}^{\lfloor\log L(p)\rfloor} \mathbb{P}\left(\hat{\tau}_{2}<p ; \hat{R}_{1} \in\left[\frac{L(p)}{2^{k+1}}, \frac{L(p)}{2^{k}}\right)\right) .
\end{aligned}
$$

We show that there is a universal constant $C_{1}$ such that for any $p>p_{c}$ and $m \leq$ $L(p) / 2$,

$$
\mathbb{P}\left(\hat{\tau}_{2}<p ; \hat{R}_{1} \in[m, 2 m]\right) \leq C_{1} \mathbb{P}_{c r}(0 \leftrightarrow \partial B(L(p)))
$$

From [20], $\mathbb{P}\left(\hat{R}_{1} \geq L(p)\right) \leq C_{2} \mathbb{P}_{c r}(0 \leftrightarrow \partial B(L(p)))$. Therefore the upper bound in (3.1) will immediately follow from (3.2). We partition the event $\left\{\hat{\tau}_{2}<p ; \hat{R}_{1} \in\right.$ $[m, 2 m]\}$ according to the value of $\hat{\tau}_{1}$ :

$$
\sum_{j=1}^{\log ^{*} m} \mathbb{P}\left(\hat{\tau}_{2}<p ; \hat{R}_{1} \in[m, 2 m] ; \hat{\tau}_{1} \in\left[p_{m}(j), p_{m}(j-1)\right)\right) .
$$

Note that if the event $\left\{\hat{R}_{1} \geq m, \hat{\tau}_{1} \in\left[p_{m}(j), p_{m}(j-1)\right)\right\}$ occurs then (a) there is a $p_{m}(j-1)$-open path from the origin to $\partial B(m)$, and (b) the origin is surrounded by a $p_{m}(j)$-closed circuit of diameter at least $m$ in the dual lattice. Also note that if the event $\left\{\hat{\tau}_{2}<p, \hat{R}_{1} \leq 2 m\right\}$ occurs then there is a $p$-open path from $B(2 m)$ to $\partial B(L(p))$. by

From the above observations, it follows that the sum (3.3) is bounded from above

$$
\sum_{j=1}^{\log ^{*} m} \mathbb{P}\left(0 \stackrel{p_{m}(j-1)}{\longleftrightarrow} \partial B(m) ; B(2 m) \stackrel{p}{\longleftrightarrow} \partial B(L(p)) ; B_{m, p_{m}(j)}\right) .
$$

The FKG inequality and independence give an upper bound of

$$
\sum_{j=1}^{\log ^{*} m} \mathbb{P}_{p_{m}(j-1)}(0 \leftrightarrow \partial B(m)) \mathbb{P}_{p}(B(2 m) \leftrightarrow \partial B(L(p))) \mathbb{P}_{p_{m}(j)}\left(B_{m}\right) .
$$

It follows from (2.4) and (2.8) that $\mathbb{P}_{p_{m}(j)}\left(B_{m}\right) \leq C_{3}(\log (j-1) m)^{-C_{4}}$ for some $C_{3}$ and $C_{4}$, where $C_{4}$ can be made arbitrarily large given that $C_{*}$ is made large enough. Inequalities (2.7) and (2.5) give

$$
\mathbb{P}_{p_{m}(j-1)}(0 \leftrightarrow \partial B(m)) \leq C_{5}(\log (j-1) m)^{\frac{1}{2}} \mathbb{P}_{c r}(0 \leftrightarrow \partial B(m)),
$$


and (2.5) and the RSW Theorem give

$$
\mathbb{P}_{p}(B(2 m) \leftrightarrow \partial B(L(p))) \leq C_{6} \mathbb{P}_{c r}(B(2 m) \leftrightarrow \partial B(L(p)))
$$

Also, the RSW Theorem and the FKG inequality imply that

$$
\mathbb{P}_{c r}(0 \leftrightarrow \partial B(m)) \mathbb{P}_{c r}(B(2 m) \leftrightarrow \partial B(L(p))) \leq C_{7} \mathbb{P}_{c r}(0 \leftrightarrow \partial B(L(p))) .
$$

Therefore, we obtain that the probability $\mathbb{P}\left(\hat{\tau}_{2}<p ; \hat{R}_{1} \in[m, 2 m]\right)$ is bounded from above by

$$
C_{8} \mathbb{P}_{c r}(0 \leftrightarrow \partial B(L(p))) \sum_{j=1}^{\log ^{*} m}\left(\log ^{(j-1)} m\right)^{-C_{4}+1 / 2}
$$

As in $[10,(2.26)]$, one can easily show that, for $C_{4}>1$,

$$
\sum_{j=1}^{\log ^{*} m}(\log (j-1) m)^{-C_{4}+1 / 2}<C_{9}
$$

The upper bound in (3.1) follows.

We now prove the lower bound in (1.1). For $p>p_{c}$ and a positive integer $m<$ $L(p) / 2$, we consider the event $C_{m, p}$ that there exists an edge $e \in \operatorname{Ann}(m, 2 m)$ such that

$-\tau_{e} \in\left(p_{c}, p_{m}\right)$

- there exist two $p_{c}$-open paths in $B(2 L(p)) \backslash\{e\}$, one connecting the origin to one of the endpoints of $e$, and another connecting the other endpoint of $e$ to the boundary of $B(2 L(p))$;

- there exists a $p_{m}$-closed dual path $P$ in $\operatorname{Ann}(m, 2 m)^{*} \backslash\left\{e^{*}\right\}$ connecting the endpoints of $e^{*}$ such that $P \cup\left\{e^{*}\right\}$ is a circuit around the origin;

- there exists a $p_{c}$-open circuit around the origin in $\operatorname{Ann}(L(p), 2 L(p))$;

- there exists a $p$-open path connecting $B(L(p))$ to infinity.

See Fig. 1 for an illustration of the event $C_{m, p}$. It can be shown similarly to [4, Corollary 6.2] that

$$
\mathbb{P}\left(C_{m, p}\right) \geq C_{10} \mathbb{P}_{c r}(0 \leftrightarrow \partial B(L(p))),
$$

where we also use the fact that $\mathbb{P}_{p}(B(L(p)) \leftrightarrow \infty)>C_{11}$ [see (2.6)]. It remains to notice that for fixed $p$, the events $C_{\left\lfloor L(p) / 2^{k}\right\rfloor, p}$ are disjoint and each of them implies the event $\left\{\hat{\tau}_{2}<p\right\}$. Therefore,

$$
\mathbb{P}\left(\hat{\tau}_{2}<p\right) \geq \sum_{k=0}^{\lfloor\log L(p)\rfloor-1} \mathbb{P}\left(C_{\left\lfloor L(p) / 2^{k}\right\rfloor, p}\right) \geq C_{10}\lfloor\log L(p)\rfloor \mathbb{P}_{c r}(0 \leftrightarrow \partial B(L(p))) .
$$




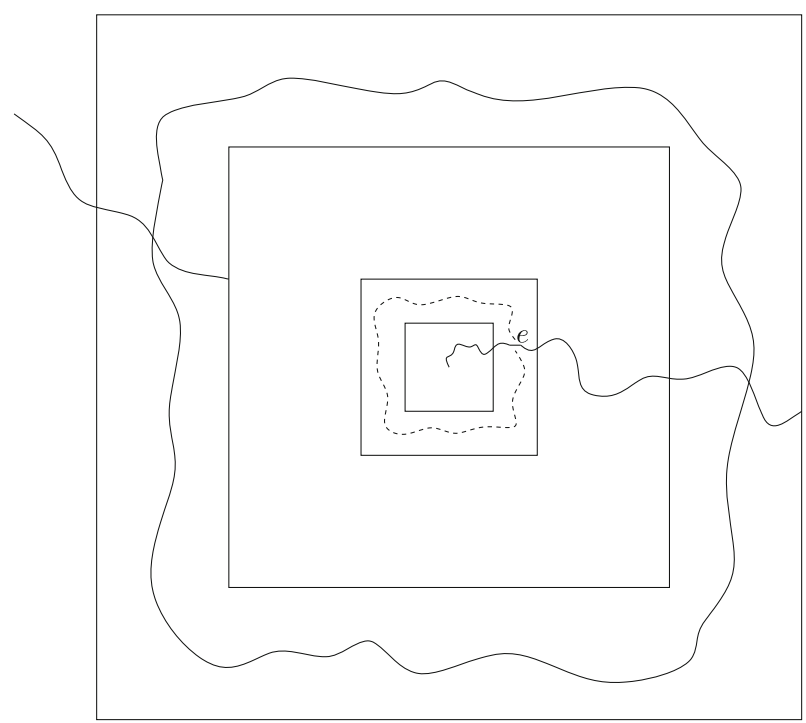

Fig. 1 The event $C_{m, p}$. The boxes, in order from smallest to largest, are $B(m), B(2 m), B(L(p))$, and $B(2 L(p))$. The dotted path is $p_{m}$-closed, the path to infinity is $p_{m}$-open, and all other paths are $p_{c}$-open

\section{Proof of Theorem 1.2}

The case $k=1$ is considered in [20, Theorem 2]. We give the proof for $k=2$. The proof for $k \geq 3$ is similar to the proof for $k=2$, and we omit the details.

We first prove the upper bound. By the RSW Theorem, it is sufficient to bound the probability $\mathbb{P}\left(\left|\hat{V}_{2}\right| \geq 2 n^{2} \pi_{n}\right)$. We partition this probability according to the value of the radii $\hat{R}_{1}$ and $\hat{R}_{2}$, defined in Sect. 1.2. Without loss of generality we can assume that $n=2^{N}$.

$$
\begin{aligned}
\mathbb{P}\left(\left|\hat{V}_{2}\right| \geq 2 n^{2} \pi_{n}\right) \leq & \mathbb{P}\left(\hat{R}_{2} \geq n\right) \\
& +\sum_{m=1}^{N} \sum_{k=1}^{m} \mathbb{P}\left(\left|\hat{V}_{2}\right| \geq 2 n^{2} \pi_{n} ; \hat{R}_{1} \in\left[2^{k-1}, 2^{k}\right) ; \hat{R}_{2} \in\left[2^{m-1}, 2^{m}\right)\right) .
\end{aligned}
$$

It follows from [4] that $\mathbb{P}\left(\hat{R}_{2} \geq n\right) \asymp(\log n) \pi_{n}$. We now consider the second term. We decompose the probability of the event

$$
E_{n, k, m}=\left\{\left|\hat{V}_{2}\right| \geq 2 n^{2} \pi_{n} ; \hat{R}_{1} \in\left[2^{k-1}, 2^{k}\right) ; \hat{R}_{2} \in\left[2^{m-1}, 2^{m}\right)\right\}
$$

according to the values of $\hat{\tau}_{1}$ and $\hat{\tau}_{2}$ :

$$
\sum_{i=1}^{\log * 2^{k}} \sum_{j=1}^{\log * 2^{m}} \mathbb{P}\left(E_{n, m, k} ; \hat{\tau}_{1} \in\left[p_{2^{k}}(i), p_{2^{k}}(i-1)\right) ; \hat{\tau}_{2} \in\left[p_{2^{m}}(j), p_{2^{m}}(j-1)\right)\right) .
$$


We consider the event $D_{n, k, m}$ that the number of vetices in the annulus $\operatorname{Ann}\left(2^{k}, 2^{m}\right)$ connected to $B\left(2^{k}\right)$ inside $A n n\left(2^{k}, 2^{m}\right)$ is at least $n^{2} \pi_{n}$. If the vertices in the definition of $D_{n, k, m}$ are connected to $B\left(2^{k}\right)$ by $p$-open paths, we denote the corresponding event by $D_{n, k, m}(p)$. We also consider the event $D_{n, k}$ that the number of vertices in the box $B\left(2^{k}\right)$ connected to the boundary $\partial B\left(2^{k}\right)$ is at least $n^{2} \pi_{n}$. If the vertices in the definition of $D_{n, k}$ are connected to $\partial B\left(2^{k}\right)$ by $p$-open paths, we denote the corresponding event by $D_{n, k}(p)$. The probability of a typical summand in (4.1) can be bounded from above by

$$
\mathbb{P}\left(\begin{array}{c}
B_{2^{k-1}, p_{2^{k}}(i)} ; B_{2^{m-1}, p_{2^{m}}(j)} ; 0 \stackrel{p_{2^{k}}(i-1)}{\longleftrightarrow} \partial B\left(2^{k}\right) ; B\left(2^{k}\right) \stackrel{p_{2^{m}(j-1)}}{\longleftrightarrow} \infty ; \\
D_{n, k, m}\left(p_{2^{m}}(j-1)\right) \cup D_{n, k}\left(p_{2^{k}}(i-1)\right)
\end{array}\right),
$$

where we use the fact that $\hat{\tau}_{1}>\hat{\tau}_{2}$ a.s.

We use the FKG inequality and independence to estimate the above probability. It is no greater than

$$
\begin{aligned}
& \mathbb{P}\left(B_{2^{k-1}, p_{2^{k}}(i)} ; B_{2^{m-1}, p_{2^{m}}(j)}\right) \mathbb{P}_{p_{2^{k}}(i-1)}\left(0 \leftrightarrow \partial B\left(2^{k}\right)\right) \mathbb{P}_{p_{2^{m}}(j-1)} \\
& \quad \times\left(B\left(2^{k}\right) \leftrightarrow \infty ; D_{n, k, m}\right) \\
& \quad+\mathbb{P}\left(B_{2^{k-1}, p_{2^{k}}(i)} ; B_{2^{m-1}, p_{2^{m}}(j)}\right) \mathbb{P}_{p_{2^{k}}(i-1)}\left(0 \leftrightarrow \partial B\left(2^{k}\right) ; D_{n, k}\right) \mathbb{P}_{p_{2^{m}}(j-1)} \\
& \quad \times\left(B\left(2^{k}\right) \leftrightarrow \infty\right)
\end{aligned}
$$

The probability $\mathbb{P}\left(B_{2^{k-1}, p_{2^{k}}(i)} ; B_{2^{m-1}, p_{2^{m}}(j)}\right)$ is bounded from above by [4, (6.6)]

$$
C_{1}\left(\log (i-1) 2^{k}\right)^{-C_{2}}\left(\log (j-1) 2^{m}\right)^{-C_{2}},
$$

where the constant $C_{2}$ can be made arbitrarily large given $C_{*}$ is made large enough.

We first estimate (4.2). It follows from (2.7) that

$$
\begin{aligned}
\mathbb{P}_{p_{2^{k}}(i-1)}\left(0 \leftrightarrow \partial B\left(2^{k}\right)\right) \leq & C_{3}\left(\log ^{(i-1)} 2^{k}\right)^{1 / 2} \mathbb{P}_{c r}\left(0 \leftrightarrow \partial B\left(2^{k}\right)\right) \\
\leq & C_{4}\left(\log ^{(i-1)} 2^{k}\right)^{1 / 2}\left(\log ^{(j-1)} 2^{m}\right)^{1 / 2} \\
& \times \frac{\mathbb{P}_{c r}\left(0 \leftrightarrow \partial B\left(2^{m}\right)\right)}{\mathbb{P}_{p_{2^{m}}(j-1)}\left(B\left(2^{k}\right) \leftrightarrow \infty\right)} .
\end{aligned}
$$

Substitution gives the following upper bound for (4.2):

$$
\begin{aligned}
& \mathbb{P}_{c r}\left(0 \leftrightarrow \partial B\left(2^{m}\right)\right) C_{1} C_{4}\left(\log { }^{(i-1)} 2^{k} \log (j-1) 2^{m}\right)^{-C_{2}+1 / 2} \mathbb{P}_{p_{2^{m}}(j-1)} \\
& \times\left(D_{n, k, m} \mid B\left(2^{k}\right) \leftrightarrow \infty\right) .
\end{aligned}
$$

We now estimate (4.3). It follows from the FKG inequality and (2.7) that

$$
\mathbb{P}_{p_{2^{k}}(i-1)}\left(0 \leftrightarrow \partial B\left(2^{k}\right)\right) \leq C_{5}\left(\log { }^{(i-1)} 2^{k}\right)^{1 / 2} \mathbb{P}_{c r}\left(0 \leftrightarrow \partial B\left(2^{k}\right)\right)
$$


Substitution gives the following upper bound for (4.3):

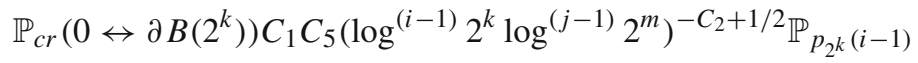

$$
\begin{aligned}
& \times\left(D_{n, k} \mid 0 \leftrightarrow \partial B\left(2^{k}\right)\right) .
\end{aligned}
$$

Therefore, the sum (4.1) is bounded from above by

$$
\begin{aligned}
& C_{6} \mathbb{P}_{c r}\left(0 \leftrightarrow \partial B\left(2^{m}\right)\right) \sum_{i=1}^{\log 2^{k}} \sum_{j=1}^{\log * 2^{m}}\left(\log { }^{(i-1)} 2^{k} \log (j-1) 2^{m}\right)^{-C_{2}+1 / 2} \mathbb{P}_{p_{2}(j-1)} \\
& \quad \times\left(D_{n, k, m} \mid B\left(2^{k}\right) \leftrightarrow \infty\right) \\
& \quad+C_{6} \mathbb{P}_{c r}\left(0 \leftrightarrow \partial B\left(2^{k}\right)\right) \sum_{i=1}^{\log 2^{k}} \sum_{j=1}^{\log * 2^{m}}\left(\log { }^{(i-1)} 2^{k} \log (j-1) 2^{m}\right)^{-C_{2}+1 / 2} \mathbb{P}_{p_{2^{k}}(i-1)} \\
& \quad \times\left(D_{n, k} \mid 0 \leftrightarrow \partial B\left(2^{k}\right)\right) .
\end{aligned}
$$

Note that $[10,(2.26)]$ if $C_{2}>1 / 2$, then there exists $C_{7}>0$ such that for all $k$,

$$
\sum_{i=1}^{\log ^{*} 2^{k}}\left(\log (i-1) 2^{k}\right)^{-C_{2}+1 / 2} \leq C_{7}<\infty
$$

Also note that analogously to [20, Lemma 4] one can show that there exist $C_{8}-C_{11}$ such that, for all $p>p_{c}$,

$$
\mathbb{P}_{p}\left(D_{n, k, m} \mid B\left(2^{k}\right) \leftrightarrow \infty\right) \leq C_{8} \exp \left\{-C_{9} \frac{n^{2} \pi_{n}}{2^{2 m} \pi\left(2^{m}, p\right)}\right\}
$$

and

$$
\mathbb{P}_{p}\left(D_{n, k} \mid 0 \leftrightarrow \partial B\left(2^{k}\right)\right) \leq C_{10} \exp \left\{-C_{11} \frac{n^{2} \pi_{n}}{2^{2 k} \pi\left(2^{k}, p\right)}\right\},
$$

where $\pi_{n}$ and $\pi(n, p)$ are defined in Sect. 1.2. In particular,

$$
\begin{aligned}
\mathbb{P}_{p_{2^{m}}(j-1)}\left(D_{n, k, m} \mid B\left(2^{k}\right) \leftrightarrow \infty\right) & \leq C_{8} \exp \left\{-C_{9} \frac{n^{2} \pi_{n}}{2^{2 m} \pi\left(2^{m}, p_{2^{m}}(j-1)\right)}\right\} \\
& \leq C_{8} \exp \left\{-C_{12} \frac{n^{2} \pi_{n}}{2^{2 m} \pi_{2^{m}}}\left(\log (j-1) 2^{m}\right)^{-1 / 2}\right\}
\end{aligned}
$$


and, similarly,

$$
\begin{aligned}
\mathbb{P}_{p^{k}(i-1)}\left(D_{n, k} \mid 0 \leftrightarrow \partial B\left(2^{k}\right)\right) & \leq C_{10} \exp \left\{-C_{11} \frac{n^{2} \pi_{n}}{2^{2 k} \pi\left(2^{k}, p_{2^{k}}(i-1)\right)}\right\} \\
& \leq C_{10} \exp \left\{-C_{13} \frac{n^{2} \pi_{n}}{2^{2 k} \pi_{2^{k}}}\left(\log ^{(i-1)} 2^{k}\right)^{-1 / 2}\right\} .
\end{aligned}
$$

Therefore, the sum $\sum_{m=1}^{N} \sum_{k=1}^{m} \mathbb{P}\left(E_{n, k, m}\right)$ is not bigger than

$$
\begin{aligned}
& C_{14}(\log n) \pi_{n} \sum_{m=1}^{N} \frac{\pi_{2^{m}}}{\pi_{n}} \sum_{j=1}^{\log 2^{m}}\left(\log (j-1) 2^{m}\right)^{-C_{2}+1 / 2} \\
& \times \exp \left\{-C_{12} \frac{n^{2} \pi_{n}}{2^{2 m} \pi_{2^{m}}}\left(\log ^{(j-1)} 2^{m}\right)^{-1 / 2}\right\} \\
& +C_{14}(\log n) \pi_{n} \sum_{k=1}^{N} \frac{\pi_{2^{k}}}{\pi_{n}} \sum_{i=1}^{\log 2^{k}}\left(\log { }^{(i-1)} 2^{k}\right)^{-C_{2}+1 / 2} \\
& \times \exp \left\{-C_{13} \frac{n^{2} \pi_{n}}{2^{2 k} \pi_{2^{k}}}\left(\log ^{(i-1)} 2^{k}\right)^{-1 / 2}\right\},
\end{aligned}
$$

where $\log n$ comes from the fact that $\sum_{k=1}^{m} 1=m \leq N=\log n$. Finally, it follows from $[20$, p. 419] that

$$
\begin{aligned}
& \sum_{m=1}^{N} \frac{\pi_{2^{m}}}{\pi_{n}} \sum_{j=1}^{\log ^{*} 2^{m}}\left(\log (j-1) 2^{m}\right)^{-C_{2}+1 / 2} \exp \left\{-C_{12} \frac{n^{2} \pi_{n}}{2^{2 m} \pi_{2^{m}}}\left(\log (j-1) 2^{m}\right)^{-1 / 2}\right\} \\
& \quad \leq C_{15}<\infty
\end{aligned}
$$

A similar bound holds for the summand (4.4). The proof for the second inequality in (1.2) is completed.

We now prove the first inequality in (1.2). For $m \leq N$, let $C_{n, m}$ be the event that there exists an edge in $\operatorname{Ann}\left(2^{m-1}, 2^{m}\right)$ such that

- its weight $\tau_{e} \in\left(p_{c}, p_{2^{m}}\right)$;

- there exist two disjoint $p_{c}$-open paths, one connecting an end of $e$ to the origin, and one connecting the other end of $e$ to $\partial B(2 n)$;

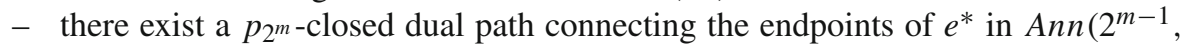
$\left.2^{m}\right)^{*}$;

- there exists a $p_{c}$-open circuit in $\operatorname{Ann}(n, 2 n)$.

It can be shown similarly to [4, Corollary 6.2] that $\mathbb{P}\left(C_{n, m}\right) \asymp \pi_{n}$. We also note that the events $C_{n, m}$ are disjoint and each of them implies the event $\left\{\hat{R}_{2} \geq n\right\}$. Using the arguments from the proof of [4, Corollary 6.2], it follows that, for any $x \in \operatorname{Ann}\left(2^{N-1}, n\right)=: A_{n}$ and $1 \leq m \leq N-2$,

$$
\mathbb{P}\left(x \stackrel{p_{c}}{\longleftrightarrow} \partial B(2 n) \mid C_{n, m}\right) \geq C_{16} \pi_{n},
$$


from which we conclude that

$$
\mathbb{E}\left(\left|\left(\hat{V}_{1} \cup \hat{V}_{2}\right) \cap A_{n}\right| \mid C_{n, m}\right) \geq C_{17} n^{2} \pi_{n} .
$$

We will show later that, for $1 \leq m \leq N-2$,

$$
\mathbb{E}\left(\left|\left(\hat{V}_{1} \cup \hat{V}_{2}\right) \cap A_{n}\right|^{2} \mid C_{n, m}\right) \leq C_{18}\left(\mathbb{E}\left(\left|\left(\hat{V}_{1} \cup \hat{V}_{2}\right) \cap A_{n}\right| \mid C_{n, m}\right)\right)^{2}
$$

If (4.6) holds, the second moment estimate gives that, for some $C_{19}>0$,

$$
\mathbb{P}\left(\left|\hat{V}_{1} \cup \hat{V}_{2}\right| \geq C_{19} n^{2} \pi_{n} ; C_{n, m}\right) \geq C_{19} \mathbb{P}\left(C_{n, m}\right) \geq C_{20} \pi_{n}
$$

Therefore

$$
\begin{aligned}
\mathbb{P}\left(\left|\hat{V}_{1} \cup \hat{V}_{2}\right| \geq C_{19} n^{2} \pi_{n} ; \hat{R}_{2} \geq n\right) & \geq \sum_{m=1}^{N-2} \mathbb{P}\left(\left|\hat{V}_{1} \cup \hat{V}_{2}\right| \geq C_{19} n^{2} \pi_{n} ; C_{n, m}\right) \\
& \geq C_{20}(N-2) \pi_{n} .
\end{aligned}
$$

In particular, using (2.7), we obtain $\mathbb{P}\left(\left|\hat{V}_{1} \cup \hat{V}_{2}\right| \geq n^{2} \pi_{n}\right) \geq C_{21}(\log n) \pi_{n}$. Recall that $\mathbb{P}\left(\left|\hat{V}_{1}\right| \geq n^{2} \pi_{n}\right) \asymp \pi_{n}$. It immediately gives the inequality $\mathbb{P}\left(\left|\hat{V}_{2}\right| \geq n^{2} \pi_{n}\right) \geq$ $C_{22}(\log n) \pi_{n}$.

It remains to prove (4.6). Note that

$$
\begin{aligned}
\mathbb{E}\left(\left|\left(\hat{V}_{1} \cup \hat{V}_{2}\right) \cap A_{n}\right|^{2} \mid C_{n, m}\right)= & \sum_{x, y \in A_{n}} \mathbb{P}\left(x, y \in \hat{V}_{1} \mid C_{n, m}\right) \\
& +\sum_{x, y \in A_{n}} \mathbb{P}\left(x, y \in \hat{V}_{2} \mid C_{n, m}\right),
\end{aligned}
$$

where we use the fact that, by construction, $\hat{V}_{1}$ and $\hat{V}_{2}$ cannot both intersect $A_{n}$. We estimate the two sums on the r.h.s. separately. We only consider the first sum. The other sum is treated similarly. We decompose the probability $\mathbb{P}\left(x, y \in \hat{V}_{1} ; C_{n, m}\right)$ according to the value of $\hat{\tau}_{1}$ :

$$
\sum_{j=1}^{\log ^{*} n} \mathbb{P}\left(x, y \in \hat{V}_{1} ; C_{n, m} ; \hat{\tau}_{1} \in\left[p_{n}(j), p_{n}(j-1)\right)\right) .
$$

Using arguments as in the first part of the proof of this theorem, the above sum is bounded from above by 


$$
\begin{aligned}
& \sum_{j=1}^{\log ^{*} n} \mathbb{P}\left(\begin{array}{c}
0 \stackrel{p_{c}}{\longleftrightarrow} \partial B\left(2^{m-1}\right) ; B\left(2^{m}\right) \stackrel{p_{c}}{\longleftrightarrow} \partial B\left(2^{N-2}\right) ; \\
x \stackrel{p_{n}(j-1)}{\longleftrightarrow} \partial B\left(x, 2^{N-2}\right) ; y \stackrel{p_{n}(j-1)}{\longleftrightarrow} \partial B\left(y, 2^{N-2}\right) ; \\
B_{n, p_{n}(j)}
\end{array}\right) \\
& \leq \mathbb{P}_{c r}\left(0 \leftrightarrow \partial B\left(2^{m-1}\right)\right) \mathbb{P}_{c r}\left(B\left(2^{m}\right) \leftrightarrow \partial B\left(2^{N-2}\right)\right) \\
& \quad \times \sum_{j=1}^{\log ^{*} n} \mathbb{P}_{p_{n}(j)}\left(B_{n}\right) \mathbb{P}_{p_{n}(j-1)}\left(x \leftrightarrow \partial B\left(x, 2^{N-2}\right) ; y \leftrightarrow \partial B\left(y, 2^{N-2}\right)\right) .
\end{aligned}
$$

Again, using tools from the first part of the proof of this theorem (see also the proof of Theorem 1.5 in [4]), the above sum is no greater than

$$
C_{23} \mathbb{P}_{c r}(0 \leftrightarrow \partial B(n)) \mathbb{P}_{c r}\left(x \leftrightarrow \partial B\left(x, 2^{N-2}\right) ; y \leftrightarrow \partial B\left(y, 2^{N-2}\right)\right) .
$$

Similar arguments apply to the second sum in (4.7). Since $\mathbb{P}\left(C_{m, n}\right) \asymp \pi_{n}$, we get

$$
\begin{aligned}
& \mathbb{E}\left(\left|\left(\hat{V}_{1} \cup \hat{V}_{2}\right) \cap A_{n}\right|^{2} \mid C_{n, m}\right) \\
& \quad \leq C_{24} \sum_{x, y \in A_{n}} \mathbb{P}_{c r}\left(x \leftrightarrow \partial B\left(x, 2^{N-2}\right), y \leftrightarrow \partial B\left(y, 2^{N-2}\right)\right) .
\end{aligned}
$$

The last sum is bounded from above by $C_{25} n^{4} \pi_{n}^{2}$ (see, e.g., the proof of Theorem 8 in [12]), which along with (4.5) gives (4.6).

\section{Proof of Theorems 1.3-1.5}

\subsection{Proof of Theorem 1.3}

We will use the following lemma. For $m, n \geq 1$, and $p \in[0,1]$, let $N(m, n, p)$ be the number of edges $e$ in the annulus $\operatorname{Ann}(n, 2 n)$ such that (a) $e$ is connected to $\partial B\left(e_{x}, m\right)$ by two disjoint $p$-open paths, (b) $e^{*}$ is connected to $\partial B\left(e_{x}, m\right)^{*}$ by two disjoint $p_{c}$-closed paths, (c) the open and closed paths are disjoint and alternate, and (d) $\tau_{e} \in\left[p_{c}, p\right]$.

Lemma 5.1 Let $m$ be such that $m \leq L(p)$ and $m \leq n$. There exists $C_{1}$ such that for all $t, n$,

$$
\mathbb{E}\left(N(m, n, p)^{t}\right) \leq t !\left(C_{1} \frac{n}{m}\right)^{2 t} .
$$

Proof The proof is very similar to the proof of the upper bound in [12, Theorem 8], where we need to use [4, Lemma 6.3] to deal with $p$-open paths. We omit the details.

To continue the proof of Theorem 1.3, define for $n \geq 1$ and $k$ with $0 \leq k \leq \log ^{*} n$, the event

$$
H_{n, k}=\left\{\begin{array}{c}
\text { There exists a } p_{n}(k)-\text { open circuit in } A n n(n / 4, n / 2) \\
\text { which is connected to infinity by a } p_{n}(k)-\text { open path. }
\end{array}\right\}
$$


where $p_{n}(k)$ is defined in (2.3). Let us decompose the $t$ th moment of $O(n, 2 n)$ according to the events $H_{n, k}$. By (2.4) and (2.8), there exists $C_{2}, C_{3}$ such that for all $n, k$,

$$
\mathbb{P}\left(H_{n, k}^{c}\right) \leq C_{2}\left(\log ^{(k-1)} n\right)^{-C_{*} C_{3}} .
$$

Writing $n_{k}=\frac{n}{C_{*} \log ^{(k)} n}$ and using the Cauchy-Schwarz inequality for $1<k<\log ^{*} n$,

$$
\begin{aligned}
\mathbb{E}\left(O(n, 2 n)^{t} ; H_{n, k}, H_{n, k+1}^{c}\right) & \leq\left(\mathbb{E}\left(N\left(n_{k}, n, p_{n}(k)\right)\right)^{2 t}\right)^{1 / 2}\left(\mathbb{P}\left(H_{n, k+1}^{c}\right)\right)^{1 / 2} \\
& \leq((2 t) !)^{1 / 2}\left(C_{1} C_{*} \log ^{(k)} n\right)^{2 t} C_{2}^{1 / 2}\left(\log ^{(k)} n\right)^{-\frac{C_{*} C_{3}}{2}} \\
& =\left(C_{2}(2 t) !\right)^{1 / 2}\left(C_{*} C_{1}\right)^{2 t}\left(\log ^{(k)} n\right)^{\frac{4 t-C_{*} C_{3}}{2}} .
\end{aligned}
$$

Choosing $C_{*}=\frac{4 t+2}{C_{3}}$, this becomes

$$
\left(C_{2}(2 t) !\right)^{1 / 2}\left(\frac{(4 t+1) C_{1}}{C_{3}}\right)^{2 t}\left(\log ^{(k)} n\right)^{-1} \leq\left(C_{4} t\right)^{3 t}\left(\log ^{(k)} n\right)^{-1}
$$

for some $C_{4}$. For the case $k=0$, we have

$$
\mathbb{E}\left(O(n, 2 n)^{t} ; H_{n, 1}^{c}\right) \leq n^{2 t} \mathbb{P}\left(H_{n, 1}^{c}\right) \leq \frac{C_{2}}{n} \leq C_{2}
$$

If we sum over $k$ and bound $\sum_{k=1}^{\left(\log ^{*} n\right)-1}\left(\log ^{(k)} n\right)^{-1}$ independent of $n$ as in [10, (2.26)], we get

$$
\mathbb{E}\left(O(n, 2 n)^{t}\right) \leq(C t)^{3 t}
$$

\subsection{Proof of Theorem 1.4}

Proof of upper bound Consider the event $A$ that, for all large $n$, for all $1 \leq i \leq n$, the annulus $\operatorname{Ann}\left(2^{i}, 2^{i+c \log n}\right)$ contains a $p_{c}$-open circuit around the origin. Note that $\mathbb{P}(A)=1$ for large enough $c$. We assume that $c$ is an integer. Then $2^{c \log n}=n^{c}$ is an integer too.

In the annulus $\operatorname{Ann}\left(2^{i}, 2^{i+2 c \log n+1}\right)$, we define the graph $\mathcal{G}_{i}^{n}$ as follows. Let $\mathcal{U}$ be the union of $p_{c}$-open clusters in $A n n\left(2^{i}, 2^{i+2 c \log n+1}\right)$ attached to $\partial B\left(2^{i+2 c \log n+1}\right)$. In particular, we assume that all the sites in $\partial B\left(2^{i+2 c \log n+1}\right)$ are in $\mathcal{U}$. If $\mathcal{U}$ contains a path from $B\left(2^{i}\right)$ to $\partial B\left(2^{i+2 c \log n+1}\right)$, we define $\mathcal{G}_{i}^{n}$ as $\mathcal{U}$. Otherwise, we consider the invasion percolation cluster $\mathcal{I}$ in $\operatorname{Ann}\left(2^{i}, 2^{i+2 c \log n+1}\right)$ of the invasion percolation process with $G_{0}=B\left(2^{i}\right)$ (that is $B\left(2^{i}\right)$ is assumed to be invaded at step 0 ) terminated at the first time a site from $\mathcal{U}$ is invaded, and define $\mathcal{G}_{i}^{n}$ as $\mathcal{I} \cup \mathcal{U}$. We say that an edge $e$ is disconnecting for $\mathcal{G}_{i}^{n}$, if the graph $\mathcal{G}_{i}^{n} \backslash\{e\}$ does not contain a path from $B\left(2^{i}\right)$ to $\partial B\left(2^{i+2 c \log n+1}\right)$. 
Let $X_{i}^{n}$ be the number of disconnecting edges for $\mathcal{G}_{i}^{n}$ in $A n n\left(2^{i+c \log n}, 2^{i+c \log n+1}\right)$. Note that if the event $A$ occurs then, for all large $n, X_{i}^{n}$ dominates $O\left(2^{i+c \log n}\right.$, $\left.2^{i+c \log n+1}\right)$, the number of outlets of the IPC $\mathcal{S}$ of the origin in $\operatorname{Ann}\left(2^{i+c \log n}\right.$, $\left.2^{i+c \log n+1}\right)$. Moreover, for any $i<\lfloor 3 c \log n\rfloor,\left(X_{i+k\lfloor 3 c \log n\rfloor}^{n}\right)_{k=0}^{\lfloor n / 3 c \log n\rfloor-1}$ are independent and the reader can verify that the proof of Theorem 1.3 is valid when the number of outlets is replaced with $X_{i}^{n}$. Therefore, there exist constants $\lambda>0$ and $C_{5}<\infty$ so that, for all $n$ and $i$,

$$
\mathbb{E} \exp \left(\lambda\left(X_{i}^{n}\right)^{1 / 3}\right)<C_{5}
$$

Let $Y_{i}$ be a sequence of independent integer-valued random variables with $\mathbb{P}\left(Y_{i}>\right.$ $n)=\min \left\{1, C_{5} e^{-\lambda n^{1 / 3}}\right\}$. Then, for any $i<\lfloor 3 c \log n\rfloor,\left(X_{i+k\lfloor 3 c \log n\rfloor}^{n}\right)_{k=0}^{\lfloor n / 3 c \log n\rfloor-1}$ is stochastically dominated by $\left(Y_{k}\right)_{k=0}^{\lfloor n / 3 c \log n\rfloor-1}$. In particular,

$$
\begin{aligned}
\mathbb{P}\left(\sum_{i=1}^{n} X_{i}^{n}>C_{6} n\right) & \leq 3 c \log n \mathbb{P}\left(\sum_{i=1}^{\lfloor n / 3 c \log n\rfloor-1} Y_{i}>C_{6} n / 3 c \log n\right) \\
& \leq C_{7} \log n \exp \left(-C_{8} n^{C_{9}}\right) .
\end{aligned}
$$

The last inequality follows, for example, from [15]. Therefore, a.s., for all large $n$, $\sum_{i=1}^{n} X_{i}^{n} \leq C_{6} n$.

Note that, if the event $A$ occurs, then, for all large $n$,

$$
O\left(2^{c \log n}, 2^{n}\right) \leq \sum_{i=1}^{n} X_{i}^{n} \leq C_{6} n .
$$

Finally, since the event $A$ occurs with probability one,

$$
O\left(2^{n}\right) \leq O\left(2^{c \log n}, 2^{n}\right)+O\left(2^{c \log (c \log n)}, 2^{c \log n}\right)+\left|B\left(2^{c \log (c \log n)}\right)\right| \leq C_{10} n .
$$

Proof of lower bound For $i \geq 1$, let $G_{i}$ be the event that there is no $p_{2^{i}}$-closed dual circuit around the origin with radius larger than $2^{i+\log i}$, and let $G$ be the event that $G_{i}$ occurs for all but finitely many $i$. It is easy to see [using inequality (2.8)] that $\mathbb{P}(G)=1$.

For $i \geq 1$, let $K_{i}$ be the event that (a) there exists a $p_{2^{i}}$-closed dual circuit $\mathcal{C}$ around the origin in $\operatorname{Ann}\left(2^{i}, 2^{i+1}\right)^{*}$, (b) there exists a $p_{c}$-open circuit $\mathcal{C}^{\prime}$ around the origin in $\operatorname{Ann}\left(2^{i}, 2^{i+1}\right)$ and (c) the circuit $\mathcal{C}^{\prime}$ is connected to $\partial B\left(2^{i+\log i}\right)$ by a $p_{2^{i}}$-open path. See Fig. 2 for an illustration of the event $G_{i} \cap K_{i}$. Note that $\mathcal{C}^{\prime}$ is in $B\left(2^{i+1}\right) \cap \operatorname{ext}(\mathcal{C})$. By RSW theorem and (2.6),

$$
\mathbb{P}\left(K_{i}\right)>C_{11}>0
$$




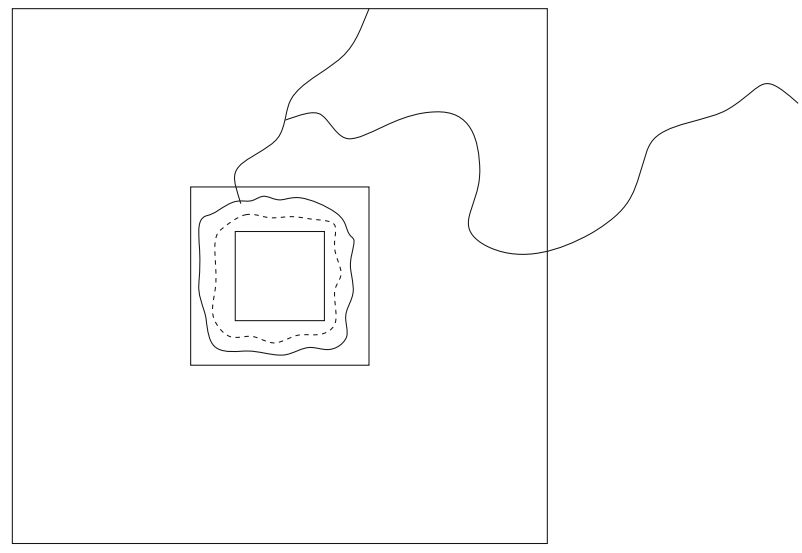

Fig. 2 The event $G_{i} \cap K_{i}$. The boxes, in order from smallest to largest, are $B\left(2^{i}\right), B\left(2^{i+1}\right)$, and $B\left(2^{i+\log i}\right)$. Because there is no $p_{2^{i}}$-closed circuit around the origin of radius larger than $2^{i+\log i}$, the $p_{2^{i}}$-open path which connects $\partial B\left(2^{i+\log i}\right)$ to the circuit in $A n n\left(2^{i}, 2^{i+1}\right)$ must be connected to $\infty$ by a $p_{2^{i}}$-open path

for some $C_{11}$ that does not depend on $i$. Fix an integer $n$, and let $j$ be an integer between 1 and $\log n$. We consider events $K_{i}^{j}=K_{j+i} \log n$. Note that, for any fixed $j$, the events $\left(K_{i}^{j}\right)_{i=0}^{(\lfloor n / \log n\rfloor)-1}$ are independent.

Let $X_{i}^{j}=I_{K_{i}^{j}}$. Recall that $\mathbb{P}\left(X_{i}^{j}=1\right)>C_{11}$. We need the following lemma. Its proof is standard, so we omit it.

Lemma 5.2 Let $c>0$. There exist $\alpha>0$ and $\beta<1$ depending on $c$ with the following property. If $X_{i}$ are independent $0 / 1$ random variables (not necessarily identically distributed) with $\mathbb{P}\left(X_{i}=1\right)>c$ for all $i$, then for all $n$,

$$
\mathbb{P}\left(\sum_{i=1}^{n} X_{i}<\alpha n\right)<\beta^{n}
$$

It follows that there exist $\alpha>0$ and $\beta<1$ such that for any $n$ and $1 \leq j \leq \log n$

$$
\mathbb{P}\left(\sum_{i=0}^{\lfloor n / \log n\rfloor-1} X_{i}^{j}<\frac{\alpha n}{\log n}\right)<\beta^{n / \log n} .
$$

Therefore,

$$
\begin{aligned}
& \mathbb{P}\left(\sum_{j=1}^{\log n} \sum_{i=0}^{\lfloor n / \log n\rfloor-1} X_{i}^{j}<\alpha n\right) \\
& \leq \mathbb{P}\left(\sum_{i=0}^{\lfloor n / \log n\rfloor-1} X_{i}^{j}<\alpha n / \log n \text { for some } j \in[1, \log n]\right) \leq \log n \beta^{n / \log n} .
\end{aligned}
$$


In particular, it follows from Borel-Cantelli's lemma that, with probability one, for all large $n$,

$$
\sum_{i=1}^{n} I_{K_{i}} \geq \alpha n .
$$

Finally, observe that the event $G$ occurs with probability one, and the event $G_{i} \cap K_{i}$ implies that there exists an outlet in $\operatorname{Ann}\left(2^{i}, 2^{i+1}\right)$. The lower bound in (1.6) follows.

Proof of Corollary 1.1 The inequalities (1.7) follow immediately from those in Theorem 1.4. Therefore we will only prove (1.8). First we show the upper bound.

Choose $c_{5}$ from (1.7). Using (2.4) and (2.8), we can show that if $C_{*}$ is made sufficiently large, then with probability one, for all large $n$, after the invasion has reached $\partial B(n)$, the weight of each further accepted edge is no larger than $p_{n}(1)$, where $p_{n}(1)$ is defined in (2.3). Therefore, for all large $k$,

$$
\hat{\tau}_{k}-p_{c} \leq p_{\left(c_{5}\right)^{k}}(1)-p_{c}
$$

Since there exists $C_{12}, C_{13}>0$ such that for all $n, p_{n}(1)-p_{c} \leq C_{12} n^{-C_{13}}$ [use (2.9)] and the fact that the 4-arm exponent is strictly smaller than 2 (see, e.g., Section 6.4 in [21]), we have

$$
\hat{\tau}_{k}-p_{c} \leq C_{12}\left(c_{5}\right)^{-C_{13} k}
$$

proving the upper bound. To show the lower bound, choose $c_{6}$ from (1.7). For $a<1$, we obtain

$$
\begin{aligned}
\mathbb{P}\left(\hat{\tau}_{k}<p_{c}+a^{k}, \hat{R}_{k}<\left(c_{6}\right)^{k}\right) & \leq \mathbb{P}\left(B\left(\left(c_{6}\right)^{k}\right) \stackrel{p_{c}+a^{k}}{\longleftrightarrow} \infty\right) \\
& \leq C_{14} \mathbb{P}_{c r}\left(B\left(\left(c_{6}\right)^{k}\right) \leftrightarrow \partial B\left(L\left(p_{c}+a^{k}\right)\right)\right) \\
& \leq C_{15}\left(\frac{\left(c_{6}\right)^{k}}{a^{-C_{16} k}}\right)^{C_{17}} \leq C_{18} e^{-C_{19} k}
\end{aligned}
$$

for constants $C_{14}-C_{19}$, where the last inequality holds for small enough $a$. The second inequality follows from (2.5). The third one follows from, for example, [9, eq. 11.90] and the fact that $L(p)>\left(p-p_{c}\right)^{-\delta}$ for some $\delta>0$ (see, e.g., Cor. 1 and eq. 2.3 from [13]). Borel-Cantelli's lemma gives the lower bound of (1.8).

\subsection{Proof of Theorem 1.5}

Given any nonempty subinterval of $(0,1]$, we will show that with probability one, $\left(\frac{\hat{\tau}_{k+1}-p_{c}}{\hat{\tau}_{k}-p_{c}}\right)$ is in this subinterval for infinitely many $k$. We will use the following fact. From [13, (4.35)] it follows that, for any $a>0$,

$$
L\left(p_{c}+a \delta\right) \asymp L\left(p_{c}+\delta\right) .
$$




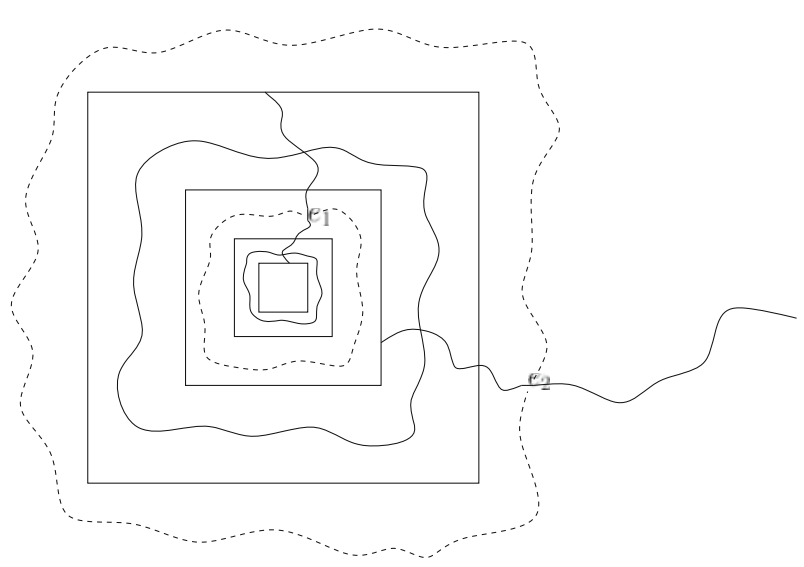

Fig. 3 The event $D_{n}$. The boxes, from smallest to largest, are $B(n), B(2 n), B(4 n)$, and $B(8 n)$. The edge $e_{1}$ is connected to both $B(n)$ and $\partial B(4 n)$. The edge $e_{2}$ is connected to both $B(4 n)$ and infinity. The solid curves represent occupied paths and the dotted curves represent vacant dual paths. In the figure, both $e_{1}$ and $e_{2}$ are outlets of the invasion

The constants above depend on $a$ but do not depend on $\delta$ so long as $\delta$ is sufficiently small.

Pick a nonempty interval $[x, y] \subset(0,1]$ and choose $a, b>0$ such that

$$
a<b \text { and } 1 \leq b x<a y .
$$

We consider the event $D_{n}$ that there exist $p_{c}$-open circuits around the origin in the annulus $A n n(n, 2 n)$; and in the annulus $A(4 n, 8 n)$, and there exist two edges, $e_{1} \in \operatorname{Ann}(2 n, 3 n)$ and $e_{2} \in A n n(8 n, 9 n)$ such that

- there is a $p_{c}$-open path connecting one of the ends of $e_{1}$ to $B(n)$, and there is a $p_{c}$-open path connecting the other end of $e_{1}$ to $\partial B(8 n)$;

- there is a $p_{c}$-open path connecting one of the ends of $e_{2}$ to $B(4 n)$, and there is a $p_{n}$-open path connecting the other end of $e_{2}$ to infinity;

- there is a $\left(p_{c}+b\left(p_{n}-p_{c}\right)\right)$-closed path $P_{1}$ in the dual lattice inside $A n n(2 n, 3 n)^{*}$ connecting the ends of $e_{1}^{*}$ such that $P_{1} \cup\left\{e_{1}^{*}\right\}$ is a circuit around the origin;

- there is a $\left(p_{c}+a y\left(p_{n}-p_{c}\right)\right)$-closed path $P_{2}$ in the dual lattice inside $A n n(8 n, 9 n)^{*}$ connecting the ends of $e_{2}^{*}$ such that $P_{2} \cup\left\{e_{2}^{*}\right\}$ is a circuit around the origin;

- the weight $\tau_{e_{1}} \in\left(p_{c}+a\left(p_{n}-p_{c}\right), p_{c}+b\left(p_{n}-p_{c}\right)\right)$, and the weight $\tau_{e_{2}} \in$ $\left(p_{c}+b x\left(p_{n}-p_{c}\right), p_{c}+a y\left(p_{n}-p_{c}\right)\right)$.

See Fig. 3 for an illustration of the event $D_{n}$. By RSW arguments and [4, Lemma 6.3] (similar to the proof of [4, Corollary 6.2]), there exists a constant $C_{20}>0$ which depends on $a, b, x$, and $y$ but not on $n$ such that

$$
\mathbb{P}\left(D_{n}\right) \geq C_{20}
$$

Since $\lim \sup _{n} D_{n}$ does not depend on the states of finitely many edges, $\mathbb{P}$ (lim sup $n$ $\left.D_{n}\right) \in\{0,1\}$. Assume that this probability is 0 . Then there exists $N$ (deterministic) 
such that

$$
\mathbb{P}\left(D_{n} \text { occurs for some } n \geq N\right)<C_{20} / 2 \text {. }
$$

But this probability is, in fact, at least $\mathbb{P}\left(D_{N}\right)$. This contradicts (5.4). Therefore

$$
\mathbb{P}\left(\lim \sup _{n} D_{n}\right)=1
$$

Note that the event $D_{n}$ implies that there exists $k=k(n)$ such that $e_{1}$ and $e_{2}$ are respectively the $k$ th and $(k+1)$ st outlets of the invasion. In particular, using the above bounds for $\tau_{e_{1}}$ and $\tau_{e_{2}}, \frac{\hat{\tau}_{k+1}-p_{c}}{\hat{\tau}_{k}-p_{c}} \in[x, y]$. Combining this with (5.5), we get $\mathbb{P}\left(\frac{\hat{\tau}_{k+1}-p_{c}}{\hat{\tau}_{k}-p_{c}} \in[x, y]\right.$ for infinitely many $\left.k\right)=1$. This completes the proof.

\section{Proof of Theorem 1.6}

Since the proof is very similar to the proof of Theorem 3 in [12], we only sketch the main ideas. From now on we fix $\sigma \in\{\text { open, closed }\}^{2 m}$, and assume that $\sigma$ consists of $m$ 'open' and $m$ 'closed'.

The RSW theorem implies that there exists $\delta>0$ such that for all $N$,

$$
\mathbb{P}_{c r}(\text { there exists an occupied circuit in } \operatorname{Ann}(N, 2 N)) \geq \delta \text {. }
$$

Since events depending on the state of edges in disjoint annuli are independent, we can find an increasing sequence $N_{i}$ such that

$$
\alpha_{i}=\mathbb{P}_{c r}\left(\text { there exists an occupied circuit in } \operatorname{Ann}\left(N_{i}, N_{i+1}\right)\right) \rightarrow 1,
$$

as $i \rightarrow \infty$. We fix the sequence $N_{i}$ and write $A_{i}$ for $\operatorname{Ann}\left(N_{i}, N_{i+1}\right)$.

Let $\mathcal{C}$ be a (self-avoiding) circuit in $\left(\mathbb{Z}^{2}, \mathbb{E}^{2}\right)$. We say that $\mathcal{C}$ is occupied with $m$ defects if all but $m$ edges of $\mathcal{C}$ are occupied. Write $F_{i}^{(m)}$ for the event that there exists an occupied circuit with $m$ defects in $A_{i}$. On the event $B\left(N_{i}\right) \leftrightarrow_{\sigma} \partial B\left(N_{i+1}\right)$, define $F_{i}\left(\mathcal{C}_{e_{1}, \ldots, e_{m}}\right)$ as the event that there exists an occupied circuit with $m$ defects in $A_{i}$ around $B\left(N_{i}\right)$, and moreover, the innermost such circuit is $\mathcal{C}$ with defected edges $e_{1}, \ldots, e_{m}$. Recall from Section 1.3.4 that the number $l$ is defined so that $|\partial B(l)| \geq|\sigma|$. Let $E$ be any event depending only on the state of edges in $B(r)$ (where we assume that $r>l$ ) and let $i$ be such that $r<N_{i}<N_{i+1}<n$. Then

$$
\begin{aligned}
\mathbb{P}_{c r}\left(E \cap\left\{B(l) \leftrightarrow{ }_{\sigma} \partial B(n)\right\}\right)= & \mathbb{P}_{c r}\left(E \cap\left\{B(l) \leftrightarrow_{\sigma} \partial B(n)\right\} \cap\left(F_{i}^{(m)}\right)^{c}\right) \\
& +\sum_{\mathcal{C} \subset A_{i}} \sum_{e_{1}, \ldots, e_{m} \in \mathcal{C} \cap \mathbb{E}^{2}} \mathbb{P}_{c r}\left(E \cap\left\{B(l) \leftrightarrow \leftrightarrow_{\sigma} \partial B(n)\right\} \cap F_{i}\left(\mathcal{C}_{e_{1}, \ldots, e_{m}}\right)\right) .
\end{aligned}
$$

Let $\left\{B(l) \leftrightarrow_{\sigma} \mathcal{C}_{e_{1}, \ldots, e_{m}}\right\}$ denote the event that $B(l)$ is $\sigma$-connected to $\mathcal{C}$ so that the $m$ disjoint closed dual paths connect $B(l)^{*}$ to the edges $e_{1}^{*}, \ldots, e_{m}^{*}$ in the interior of $\mathcal{C}$. Similarly, let $\left\{\mathcal{C}_{e_{1}, \ldots, e_{m}} \leftrightarrow_{\sigma} \partial B(n)\right\}$ denote the event that $\mathcal{C}$ is $\sigma$-connected to $\partial B(n)$ 
so that the $m$ disjoint closed dual paths connect $\partial B(n)^{*}$ to the edges $e_{1}^{*}, \ldots, e_{m}^{*}$ in the exterior of $\mathcal{C}$.

We now estimate the probability $\mathbb{P}_{c r}\left(E \cap\left\{B(l) \leftrightarrow_{\sigma} \partial B(n)\right\} \cap\left(F_{i}^{(m)}\right)^{c}\right)$. By Menger's theorem [6, Theorem 3.3.1], the event $\left\{B(l) \leftrightarrow_{\sigma} \partial B(n)\right\} \cap\left(F_{i}^{(m)}\right)^{c}$ implies that there exist $(m+1)$ disjoint closed crossings of the annulus $A_{i}$. We use Reimer's inequality [19] to conclude that the probability $\mathbb{P}_{c r}\left(E \cap\left\{B(l) \leftrightarrow \leftrightarrow_{\sigma} \partial B(n)\right\} \cap\left(F_{i}^{(m)}\right)^{c}\right)$ is bounded from above by

$$
\begin{aligned}
& \mathbb{P}_{c r}\left(B(l) \leftrightarrow_{\sigma} \partial B(n)\right) \mathbb{P}_{c r}\left(\text { there exists a closed crossing of } A_{i}\right) \\
& \quad \leq\left(1-\alpha_{i}\right) \mathbb{P}_{c r}\left(B(l) \leftrightarrow_{\sigma} \partial B(n)\right) .
\end{aligned}
$$

We have just shown how a statement similar to (17) in [12] is obtained. An analogous statement to (18) in [12] is also valid. The remainder of the proof is similar to the proof of Kesten [12], where in the proof of the statement analogous to Lemma 23 in [12] we use extensions of arm separation techniques from [18, Section 4]. We use the following analogue of Kesten's Lemma 23.

Lemma 6.1 Consider circuits $\mathcal{C}$ in annulus $A_{i}, \mathcal{D}$ in annulus $A_{i+3}$, sets of edges $e_{1}, \ldots, e_{m}$ on $\mathcal{C}$ and $f_{1}, \ldots, f_{m}$ on $\mathcal{D}$ respectively. Let $P(\mathcal{C}, \mathcal{D})$ be the probability, conditional on the event that all edges in $\mathcal{C} \backslash\left\{e_{1}, \ldots, e_{m}\right\}$ are open and $e_{1}, \ldots, e_{m}$ are closed, that (1) there are disjoint closed dual paths from $e_{i}^{*}$ to $f_{i}^{*}$, (2) there are $m$ disjoint open paths that connect $\mathcal{C}$ to $\mathcal{D}$ such that, for any two of them, there is a closed dual path (one of the paths from (1)) between them, (3) $\mathcal{D}$ is the innermost open circuit with defects $f_{1}, \ldots, f_{m}$ in annulus $A_{i+3}$, (4) there is an open circuit with $m$ defects in annulus $A_{i+2}$. (Dependence on the edges $e_{i}$ and $f_{i}$ is suppressed in the notation.) We similarly define $\mathcal{C}^{\prime}, \mathcal{D}^{\prime}$, etc. There exists a finite constant $C_{1}$ that may depend only on $m$ (it does not depend on particular choice of circuits or defects) such that

$$
\frac{P(\mathcal{C}, \mathcal{D}) P\left(\mathcal{C}^{\prime}, \mathcal{D}^{\prime}\right)}{P\left(\mathcal{C}, \mathcal{D}^{\prime}\right) P\left(\mathcal{C}^{\prime}, \mathcal{D}\right)}<C_{1}
$$

To prove Lemma 6.1, we need the following extension of Kesten's arm separation [13, Lemmas 4 and 5]. Let $\mathcal{I}$ be a fixed partition of $\partial B(1)$ (in $\mathbb{R}^{2}$ ) into $2 m$ disjoint connected subsets $\mathcal{I}_{i}$, each of diameter at least $1 /(2 m)$ (ordered clockwise). Let $\mathcal{I}(s)$ be the corresponding partition of $\partial B(s)$ into $2 m$ disjoint connected subsets $\mathcal{I}_{i}(s)=s \mathcal{I}_{i}=\left\{s x: x \in \mathcal{I}_{i}\right\}$. Let $\mathcal{I}\left(n, n^{\prime}\right)$ be the partition of $\overline{A n n\left(n, n^{\prime}\right)}$ into $2 m$ disjoint connected subsets $\mathcal{I}_{i}\left(n, n^{\prime}\right)=\cup_{n \leq s \leq n^{\prime}} \mathcal{I}_{i}(s)$.

Lemma 6.2 (external arm separation) Let $n_{0}$ and $n$ be positive integers with $n_{0} \leq$ $n-3$. We consider a circuit $\mathcal{C}$ in $B\left(2^{n_{0}}\right)$ and a set of edges $e_{1}, \ldots, e_{m}$ on $\mathcal{C}$. Let $E\left(\mathcal{C}_{e_{1}, \ldots, e_{m}}\right)$ be the event that $(1)$ the edges in $\mathcal{C} \backslash\left\{e_{1}, \ldots, e_{m}\right\}$ are open and $e_{1}, \ldots, e_{m}$ are closed, (2) there are $m$ disjoint closed dual paths from $e_{j}^{*}$ to $\partial B\left(2^{n}\right)^{*}$, (3) there are $m$ disjoint open paths from $\mathcal{C}$ to the boundary of $B\left(2^{n}\right)$ in $\left(B\left(2^{n}\right) \backslash \operatorname{int}(C)\right) \backslash\left\{e_{1}, \ldots, e_{m}\right\}$ such that these paths alternate with the closed paths defined in (2). Let $\widetilde{E}\left(\mathcal{C}_{e_{1}, \ldots, e_{m}}\right)$ be the event that $E\left(\mathcal{C}_{e_{1}, \ldots, e_{m}}\right)$ occurs with $2 m$ paths $P_{1}, \ldots, P_{2 m}$ (ordered clockwise, all 
paths with odd indices are closed, and the ones with even indices are open) satisfying the requirement that, for all $1 \leq i \leq 2 m, P_{i} \cap \overline{\operatorname{Ann}\left(2^{n-1}, 2^{n}\right)} \subset \mathcal{I}_{i}\left(2^{n-1}, 2^{n}\right)$. Then

$$
\mathbb{P}\left(E\left(\mathcal{C}_{e_{1}, \ldots, e_{m}}\right)\right) \leq C_{2} \mathbb{P}\left(\widetilde{E}\left(\mathcal{C}_{e_{1}, \ldots, e_{m}}\right)\right),
$$

where the constant $C_{2}$ may depend on $m$ but not on $n, n_{0}$, or the choice of circuit.

Remark 8 The event $\widetilde{E}$ is reminiscent of the event $\Delta$ in [13, p. 127 and Figure 8].

Remark 9 It is actually believed [7] and is the aim of ongoing work of Garban and Pete that a much stronger statement holds: given any configuration inside $B\left(2^{n_{0}}\right)$, if we condition on the existence of $m$ open paths and $m$ closed dual paths from a neighborhood of the origin to $\partial B\left(2^{n}\right)$ and these paths are alternating, then they will be well-separated (refer to [13] for this definition) on $\partial B\left(2^{n}\right)$ with positive probability independent of $n$ and the configuration inside $B\left(2^{n_{0}}\right)$.

Lemma 6.3 (internal arm separation) Let $n$ and $n_{1}$ be positive integers with $n+3 \leq n_{1}$. Consider a circuit $\mathcal{D}$ in $B\left(2^{n_{1}}\right)^{c}$ and a set of edges $f_{1}, \ldots, f_{m}$ on $\mathcal{D}$. Let $F\left(\mathcal{D}_{f_{1}, \ldots, f_{m}}\right)$ be the event that (1) the edges in $\mathcal{D} \backslash\left\{f_{1}, \ldots, f_{m}\right\}$ are open and $f_{1}, \ldots, f_{m}$ are closed, (2) there are $m$ disjoint closed dual paths from $f_{j}^{*}$ to $B\left(2^{n}\right)^{*}$, (3) there are $m$ disjoint open paths from $\mathcal{D}$ to $B\left(2^{n}\right)$ in int(D) such that these paths alternate with the closed dual paths defined in (2). Let $\widetilde{F}\left(\mathcal{D}_{f_{1}, \ldots, f_{m}}\right)$ be the event that the event $F\left(\mathcal{D}_{f_{1}, \ldots, f_{m}}\right)$ occurs with $2 m$ paths $P_{1}, \ldots, P_{2 m}$ (ordered clockwise, all paths with odd indices are closed, and the ones with even indices are open) satisfying the requirement that, for all $1 \leq i \leq 2 m, P_{i} \cap \overline{\operatorname{Ann}\left(2^{n}, 2^{n+1}\right)} \subset \mathcal{I}_{i}\left(2^{n}, 2^{n+1}\right)$. Then

$$
\mathbb{P}\left(F\left(\mathcal{D}_{f_{1}, \ldots, f_{m}}\right)\right) \leq C_{3} \mathbb{P}\left(\widetilde{F}\left(\mathcal{D}_{f_{1}, \ldots, f_{m}}\right)\right),
$$

where the constant $C_{3}$ may depend on $m$ but not on $n, n_{1}$, or the choice of circuit.

The proofs of Lemmas 6.2 and 6.3 are similar, and we only give the proof of Lemma 6.2 here. Moreover, parts of the proof of Lemma 6.2 are similar to the proof of Lemma 4 in [13]. We will refer the reader to [13] for the proof of those parts. Before we give the proof of Lemma 6.2, we show how to deduce Lemma 6.1 from the above two lemmas. Using Lemmas 6.2, 6.3 and "gluing" arguments (see [13,18]), we prove

Lemma 6.4 For two circuits, $\mathcal{C}_{1}$ in annulus $A_{i}$ and $\mathcal{D}_{1}$ in annulus $A_{i+2}$, sets of edges $e_{1}, \ldots, e_{m}$ on $\mathcal{C}_{1}$ and $f_{1}, \ldots, f_{m}$ on $\mathcal{D}_{1}$, if $M\left(\mathcal{C}_{1}, \mathcal{D}_{1}\right)$ is the probability, conditioned on the event that all edges in $\mathcal{C}_{1} \backslash\left\{e_{1}, \ldots, e_{m}\right\}$ and in $\mathcal{D}_{1} \backslash\left\{f_{1}, \ldots, f_{m}\right\}$ are open and $e_{1}, \ldots, e_{m}, f_{1}, \ldots, f_{m}$ are closed, that there are disjoint closed dual paths from $e_{i}^{*}$ to $f_{i}^{*}$ for all $i$, and there are $m$ disjoint open paths from $\mathcal{C}_{1}$ to $\mathcal{D}_{1}$ in $\overline{\operatorname{int}\left(\mathcal{D}_{1}\right)} \backslash \operatorname{int}\left(\mathcal{C}_{1}\right)$, which alternate with the closed dual paths defined above (and similar definitions for $\mathcal{C}_{2}$ and $\mathcal{D}_{2}$ ), then

$$
\frac{M\left(\mathcal{C}_{1}, \mathcal{D}_{1}\right) M\left(\mathcal{C}_{2}, \mathcal{D}_{2}\right)}{M\left(\mathcal{C}_{1}, \mathcal{D}_{2}\right) M\left(\mathcal{C}_{2}, \mathcal{D}_{1}\right)}<C_{4},
$$

for some constant $C_{4}$ that does not depend on the particular choice of circuits or defects. (Dependence on the edges $e_{i}$ and $f_{i}$ is suppressed in the notation.) 
Proof This lemma follows from Lemmas 6.2, 6.3, the RSW theorem (Section 11.7 in [9]), and the generalized FKG inequality [13, Lemma 3]. For more details we refer the reader to the proof of (2.43) in [13].

Proof of Lemma 6.1 Consider circuits $\mathcal{C}_{1}$ in annulus $A_{i+2}, \mathcal{D}_{1}$ in $A_{i+3}$, sets of edges $g_{1}, \ldots, g_{m}$ on $\mathcal{C}_{1}$ and $h_{1}, \ldots, h_{m}$ on $\mathcal{D}_{1}$ respectively. Let $H\left(\mathcal{C}_{1}, \mathcal{D}_{1}\right)$ be the probability of the event that $(1) \mathcal{C}_{1}$ is the outermost open circuit with defects $g_{1}, \ldots, g_{m}$ in annulus $A_{i+2}$, (2) $\mathcal{D}_{1}$ is the innermost open circuit with defects $h_{1}, \ldots, h_{m}$ in annulus $A_{i+3}$, (3) there are disjoint closed dual paths from $g_{i}^{*}$ to $h_{i}^{*}$, and (4) there are $m$ disjoint open paths from $\mathcal{C}_{1}$ to $\mathcal{D}_{1}$ in $\overline{\operatorname{int}\left(\mathcal{D}_{1}\right)} \backslash \operatorname{int}\left(\mathcal{C}_{1}\right)$, which alternate with the closed dual paths defined above. (Dependence on the edges $g_{i}$ and $h_{i}$ is suppressed in the notation.)

We write

$$
P(\mathcal{C}, \mathcal{D}) P\left(\mathcal{C}^{\prime}, \mathcal{D}^{\prime}\right)=\sum_{\mathcal{C}_{1}} M\left(\mathcal{C}, \mathcal{C}_{1}\right) H\left(\mathcal{C}_{1}, \mathcal{D}\right) \sum_{\mathcal{C}_{1}^{\prime}} M\left(\mathcal{C}^{\prime}, \mathcal{C}_{1}^{\prime}\right) H\left(\mathcal{C}_{1}^{\prime}, \mathcal{D}^{\prime}\right)
$$

We then apply the previous lemma to $\mathcal{C}, \mathcal{C}_{1}, \mathcal{C}^{\prime}$ and $\mathcal{C}_{1}^{\prime}$.

Proof of Lemma 6.2 We only consider the case $m=2$. The case $m=1$ is simpler, and the general case is similar to the case $m=2$. Fix a circuit $\mathcal{C}$ in $B\left(2^{n_{0}}\right)$ and edges $e, f$ on $\mathcal{C}$, and assume that the event $E\left(\mathcal{C}_{e, f}\right)$ occurs.

We define $\gamma_{1}^{l}$ as the leftmost closed dual path from $e^{*}$ to $\partial B\left(2^{n_{0}}+1 / 2\right)$ in $B\left(2^{n_{0}}+\right.$ $1 / 2) \backslash \operatorname{int}(\mathcal{C})$, and $\gamma_{1}^{r}$ as the rightmost closed dual path from $e^{*}$ to $\partial B\left(2^{n_{0}}+1 / 2\right)$ in $B\left(2^{n_{0}}+1 / 2\right) \backslash \operatorname{int}(\mathcal{C})$. We denote the first vertex on $\partial B\left(2^{n_{0}}\right)$ to the left of $\gamma_{1}^{l}$ as $a_{1}$, and the first vertex on $\partial B\left(2^{n_{0}}\right)$ to the right of $\gamma_{1}^{r}$ as $a_{2}$. Let $\gamma_{2}^{l}$ be the leftmost open path from the right end-vertex of $e$ (using the clockwise ordering of vertices end edges on $\mathcal{C})$ to $a_{2}$. This path is necessarily contained in $B\left(2^{n_{0}}\right) \backslash \operatorname{int}(\mathcal{C})$. Let $\gamma_{4}^{r}$ be the rightmost open path from the left end-vertex of $e$ (using the clockwise ordering of vertices end edges on $\mathcal{C}$ ) to $a_{1}$ in $B\left(2^{n_{0}}\right) \backslash \operatorname{int}(\mathcal{C})$. Similarly we define $\gamma_{3}^{l}, \gamma_{3}^{r}, a_{3}, a_{4}, \gamma_{4}^{l}$ and $\gamma_{2}^{r}$ (see Fig. 4).

For $i \in\{1,2,3,4\}$, let $T_{i}$ be the piece of $\partial B\left(2^{n_{0}}\right)$ between (and including) $a_{i}$ and $a_{i+1}$ that does not contain $a_{i+2}$ or $a_{i+3}$, where we use the convention $a_{i}=a_{i-4}$ for $i>4$. Note that it is possible that $a_{2}=a_{3}$ (in which case $T_{2}=\left\{a_{2}\right\}$ ) or $a_{4}=a_{1}$ (in which case $T_{4}=\left\{a_{4}\right\}$ ); however, we necessarily have $a_{1} \neq a_{2}$ and $a_{3} \neq a_{4}$.

Let $\gamma_{i}$ be the part of $\gamma_{i}^{l} \cup \gamma_{i}^{r} \cup \mathcal{C}$ that consists of the piece of $\gamma_{i}^{l}$ from the last intersection with $\gamma_{i}^{r} \cup \mathcal{C}$, the piece of $\gamma_{i}^{r}$ from the last intersection with $\gamma_{i}^{l} \cup \mathcal{C}$, and the piece of $\mathcal{C}$ that connects the first two pieces (if the pieces are disconnected). Note that it is possible that $\gamma_{2}$ or $\gamma_{4}$ is a single point set on $\partial B\left(2^{n_{0}}\right)$, which happens if $a_{2}=a_{3}$ or $a_{4}=a_{1}$, respectively. Let $R_{i}$ denote the connected subset of $\mathbb{R}^{2}$ with the boundary that consists of $T_{i}$ and $\gamma_{i}$ (see Fig. 4). Note that these sets are disjoint. Moreover, if $\gamma_{2}$ or $\gamma_{4}$ is a single point set ( $\left\{a_{2}\right\}$ or $\left\{a_{4}\right\}$, respectively), then $R_{2}$ or $R_{4}$ is the same single point set. Let $S:=B\left(2^{n_{0}}\right)^{c} \cup R_{1} \cup R_{2} \cup R_{3} \cup R_{4}$. Note that once $\gamma_{1}, \gamma_{2}, \gamma_{3}$, and $\gamma_{4}$ are fixed, the percolation process in $S$ is still an independent Bernoulli percolation.

Let $E\left(\gamma_{1}, \ldots, \gamma_{4}\right)$ be the event that (1) $\gamma_{1}$ and $\gamma_{3}$ are connected to $\partial B\left(2^{n}\right)^{*}$ by closed dual paths $P_{1}$ and $P_{3}$ in $S$, and (2) $\gamma_{2}$ and $\gamma_{4}$ are connected to $\partial B\left(2^{n}\right)$ by open paths $P_{2}$ and $P_{4}$ in $S$. Let $\widetilde{E}\left(\gamma_{1}, \ldots, \gamma_{4}\right)$ be the event that $E\left(\gamma_{1}, \ldots, \gamma_{4}\right)$ occurs with paths 


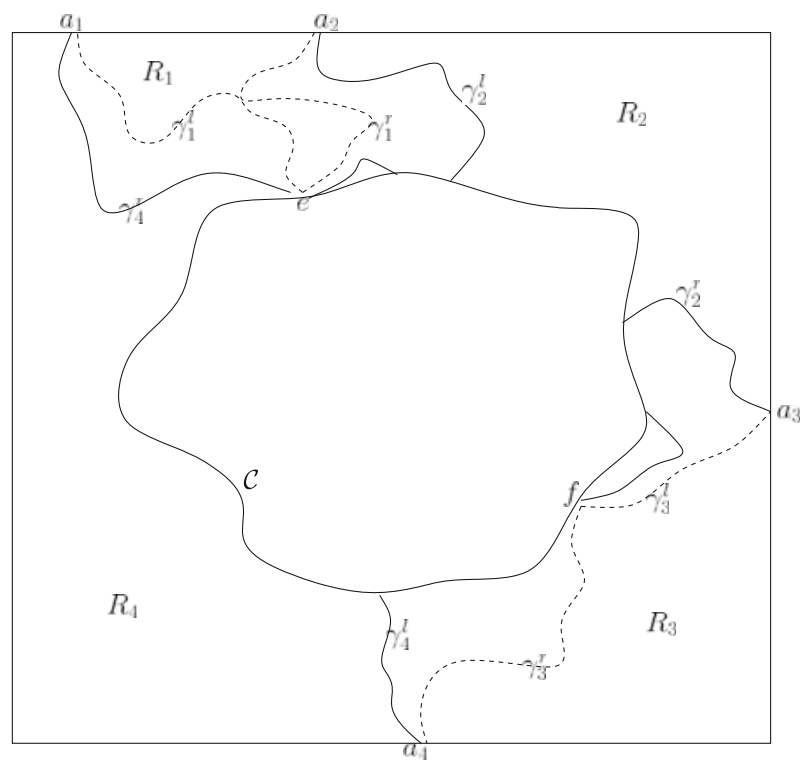

Fig. 4 The event $E\left(\mathcal{C}_{e_{1}, \ldots, e_{m}}\right)$ occurs if and only if $\gamma_{1}$ and $\gamma_{3}$ are connected by closed paths to $\partial B\left(2^{n}\right)^{*}$, and $\gamma_{2}$ and $\gamma_{4}$ are connected by open paths to $\partial B\left(2^{n}\right)$ in $B\left(2^{n_{0}}\right)^{c} \cup R_{1} \cup R_{2} \cup R_{3} \cup R_{4}$

$P_{1}, \ldots, P_{4}$ satisfying the requirement that, for all $1 \leq i \leq 4, P_{i} \cap \overline{\operatorname{Ann}\left(2^{n-1}, 2^{n}\right)} \subset$ $\mathcal{I}_{i}\left(2^{n-1}, 2^{n}\right)$. Lemma 6.2 follows if there exists a constant $C_{2}$ which does not depend on $n, n_{0}$, or the choice of $\gamma_{i}$ 's, such that

$$
\mathbb{P}\left(E\left(\gamma_{1}, \ldots, \gamma_{4}\right)\right) \leq C_{2} \mathbb{P}\left(\widetilde{E}\left(\gamma_{1}, \ldots, \gamma_{4}\right)\right)
$$

If $T_{1}, \ldots, T_{4}$ are comparable in size, the proof of (6.1) is essentially the same as the proof of Lemma 4 in [13]. If $T_{1}, \ldots, T_{4}$ are of different scales, the proof of (6.1) is similar in spirit to the proof of Lemma 4 in [13], but more involved. We indicate the differences below. We first construct a family of disjoint annuli in four stages. We define

$$
l_{i}(1)=\min \left\{l: \exists x \in 2^{l} \mathbb{Z}^{2} \cap \partial B\left(2^{n_{0}}\right) \text { s.t. } B\left(x, 2^{l}\right) \supset T_{i}\right\}
$$

if such $l$ exists (the definition implies that it is no bigger than $n_{0}$ ), and let $B_{i}(1)=$ $B\left(x_{i}(1), 2^{l_{i}(1)}\right)$ be such a box. If there are several choices for the box, we pick the first one in clockwise ordering. If there are no such $l$, we let $l_{i}(1)=n_{0}+1$ and $B_{i}(1)=B\left(2^{n_{0}+1}\right)$ (in this case $\left.x_{i}(1)=0\right)$. The boxes $B_{1}(1), \ldots, B_{4}(1)$ form a covering of $\partial B\left(2^{n_{0}}\right)$ such that $B_{i}(1) \supset T_{i}$, and either $x_{i}(1) \in T_{i}$ or $x_{i}(1)=0$ (in which case $\left.B_{i}(1)=B\left(2^{n_{0}+1}\right)\right)$. We also define

$$
\widetilde{l}_{i}(1)=\min \left\{l \geq l_{i}(1): B\left(x_{i}(1), 2^{l}\right) \supset B_{j}(1) \text { for some } j \neq i\right\} .
$$


Let $A n n_{i}(1)=A n n\left(x_{i}(1) ; 2^{l_{i}(1)}, 2^{\widetilde{l}_{i}(1)-3}\right)=B\left(x_{i}(1), 2^{\widetilde{l}_{i}(1)-3}\right) \backslash B_{i}(1)$, if $\widetilde{l}_{i}(1)-5>$ $l_{i}(1)$; otherwise, let $A n n_{i}(1)=\emptyset$. Note that if $l_{i}(1) \geq l_{i-1}(1)$ or $l_{i}(1) \geq l_{i+1}(1)$, then $A n n_{i}(1)=\emptyset$. In particular, if $l_{i}(1)=n_{0}+1$, then $A n n_{i}(1)=\emptyset$. If $A n n_{i}(1) \neq \emptyset$, we let $\widetilde{B}_{i}(1)=B\left(x_{i}(1), 2^{\widetilde{l}_{i}(1)-3}\right)$; otherwise, we let $\widetilde{B}_{i}(1)=B_{i}(1)$. We write $\widetilde{B}_{i}(1)=$ $B\left(x_{i}(1), 2^{l_{i}(1)^{\prime}}\right)$. We observe that the boxes $\widetilde{B}_{i}(1)$ form a covering of $\partial B\left(2^{n_{0}}\right)$, and that there exists $i$ such that $\left|l_{i+1}(1)^{\prime}-l_{i}(1)^{\prime}\right| \leq 5$, in other words, $\widetilde{B}_{i+1}(1)$ and $\widetilde{B}_{i}(1)$ are comparable in size. This completes the first stage of our construction.

We proceed further by defining

$$
l_{i}(2)=\min \left\{l: \exists x \in 2^{l} \mathbb{Z}^{2} \cap \partial B\left(2^{n_{0}}\right) \text { s.t. } B\left(x, 2^{l}\right) \supset\left(\widetilde{B}_{i}(1) \cup \widetilde{B}_{i+1}(1)\right)\right\}
$$

if such $l$ exists (it is necessarily not bigger than $n_{0}$ ), and let $B_{i}(2)=B\left(x_{i}(2), 2^{l_{i}(2)}\right.$ ) be such a box (if there are several choices, we pick the first one in clockwise ordering). If there are no such $l$, we let $l_{i}(2)=n_{0}+1$ and $B_{i}(2)=B\left(2^{n_{0}+1}\right)$ (in this case $\left.x_{i}(2)=0\right)$. We also define

$$
\widetilde{l}_{i}(2)=\min \left\{l \geq l_{i}(2): B\left(x_{i}(2), 2^{l}\right) \supset \widetilde{B}_{j}(1) \text { for some } j \neq i, i+1\right\} .
$$

Let $A n n_{i}(2)=A n n\left(x_{i}(2) ; 2^{l_{i}(2)}, 2^{\widetilde{l}_{i}(2)-3}\right)=B\left(x_{i}(2), 2^{\widetilde{l}_{i}(2)-3}\right) \backslash B_{i}(2)$, if $\widetilde{l}_{i}(2)-5>$ $l_{i}(2)$; otherwise, let $A n n_{i}(2)=\emptyset$. If $A n n_{i}(2) \neq \emptyset$, we define $\widetilde{B}_{i}(2)=\widetilde{B}_{i+1}(2)=$ $B\left(x_{i}(2), 2^{\tilde{l}_{i}(2)-3}\right)$. For all remaining indices $i$ for which $\widetilde{B}_{i}(2)$ is not yet defined, we let $\widetilde{B}_{i}(2)=\widetilde{B}_{i}(1)$. In other words, if we have not succeeded in building a nonempty annulus around $\widetilde{B}_{i}(1)$, we take this box unchanged to the next stage of our construction. We write $\widetilde{B}_{i}(2)=B\left(x_{i}(2), 2^{l_{i}(2)^{\prime}}\right)$. We observe that the boxes $\widetilde{B}_{i}(2)$ form a covering of $\partial B\left(2^{n_{0}}\right)$, and that there exists $i$ such that $\left|l_{i+1}(2)^{\prime}-l_{i}(2)^{\prime}\right| \leq 5$, $\left|l_{i+2}(2)^{\prime}-l_{i}(2)^{\prime}\right| \leq 5$ and $\left|l_{i+2}(2)^{\prime}-l_{i+1}(2)^{\prime}\right| \leq 5$. In other words $\widetilde{B}_{i}(2), \widetilde{B}_{i+1}(2)$ and $\widetilde{B}_{i+2}(2)$ are comparable in size.

In the third stage, for any $i$, we define

$$
l_{i}(3)=\min \left\{l: \exists x \in 2^{l} \mathbb{Z}^{2} \cap \partial B\left(2^{n_{0}}\right) \text { s.t. } B\left(x, 2^{l}\right) \supset\left(\widetilde{B}_{i}(2) \cup \widetilde{B}_{i+1}(2) \cup \widetilde{B}_{i+2}(2)\right)\right\}
$$

if such $l$ exists (it is necessarily not bigger than $n_{0}$ ), and let $B_{i}(3)=B\left(x_{i}(3), 2^{l_{i}(3)}\right)$ be such a box (if there are several choices, we pick the first one in clockwise ordering). If there are no such $l$, we let $l_{i}(3)=n_{0}+1$ and $B_{i}(3)=B\left(2^{n_{0}+1}\right)$ (in this case $\left.x_{i}(3)=0\right)$. We also define

$$
\widetilde{l}_{i}(3)=n_{0}+1
$$

Let $A n n_{i}(3)=A n n\left(x_{i}(3) ; 2^{l_{i}(3)}, 2^{\widetilde{l}_{i}(3)-3}\right)=B\left(x_{i}(3), 2^{\widetilde{l}_{i}(3)-3}\right) \backslash B_{i}(3)$, if ${\widetilde{l_{i}}}_{\underline{B}}(3)-5>$ $l_{i}(3)$, otherwise let $A \sim_{\tau_{i}}(3)=\emptyset$. If $A n n_{i}(3) \neq \emptyset$, we define $\widetilde{B}_{i}(3)=\widetilde{B}_{i+1}(3)=$ $\widetilde{B}_{i+2}(3)=B\left(x_{i}(3), 2^{\widetilde{l}_{i}(3)-3}\right)$. For all remaining indices $i$ for which $\widetilde{B}_{i}(3)$ is not yet defined, we let $\widetilde{B}_{i}(3)=\widetilde{B}_{i}(2)$. In other words, if we have not succeeded in building a nonempty annulus around $\widetilde{B}_{i}(2)$, we take this box unchanged to the next stage of our construction. We write $\widetilde{B}_{i}(3)=B\left(x_{i}(3), 2^{l_{i}(3)^{\prime}}\right)$. We observe that the boxes $\widetilde{B}_{i}(3)$ 

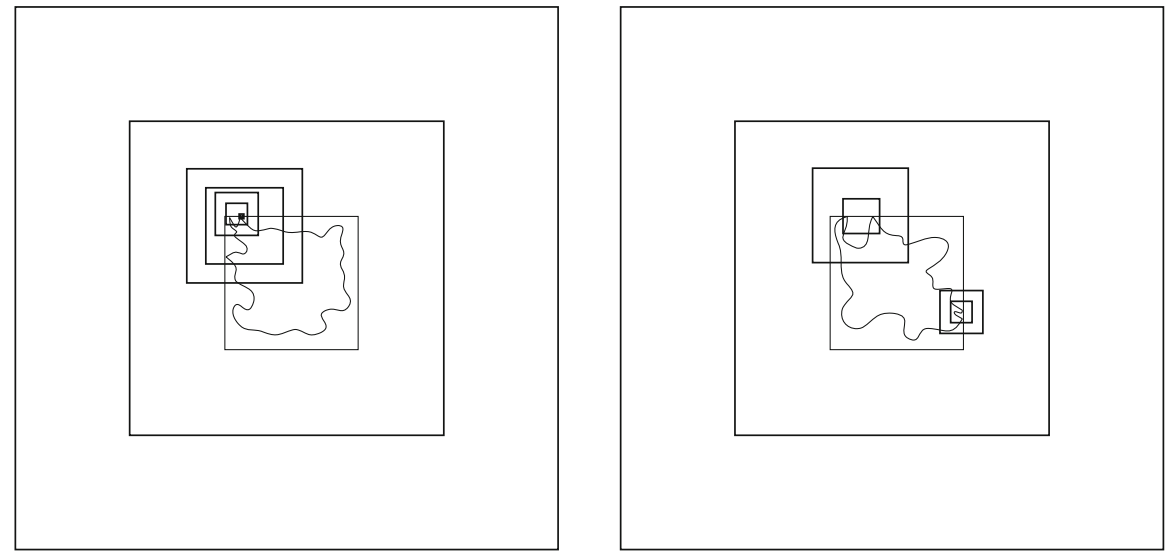

Fig. 5 The family of annuli in the left figure consists of one annulus of each level. The family of annuli in the right figure consists of two level 1 annuli and one level 4 annulus. In general, there are at most two non-empty level 1 annuli, at most one level 2, 3 or 4 annulus each

form a covering of $\partial B\left(2^{n_{0}}\right)$ such that $\left|l_{i+1}(3)^{\prime}-l_{i}(3)^{\prime}\right| \leq 5$ for all $i$. Moreover, all of these boxes are contained in $B\left(2^{n_{0}+1}\right)$.

Finally, we define the annulus $A n n_{i}(4)=A n n\left(2^{n_{0}+1}, 2^{n}\right)$.

Note that $\left(A n n_{i}(1)\right)_{i},\left(A n n_{j}(2)\right)_{j},\left(A n n_{k}(3)\right)_{k}$, and $\left(A n n_{l}(4)\right)_{l}$ are disjoint among levels and between levels. In addition, the event $E\left(\gamma_{1}, \ldots, \gamma_{4}\right)$ implies the existence of crossings of annulus $A n n_{i}(1)$ by path $P_{i}$, annulus $A n n_{i}(2)$ by paths $P_{i}$ and $P_{i+1}$, annulus $A n n_{i}$ (3) by paths $P_{i}, P_{i+1}$ and $P_{i+2}$, and annulus $A n n_{i}$ (4) by all four paths $P_{1}, \ldots, P_{4}$. Some examples of families of annuli are illustrated on Fig. 5.

To show (6.1), our strategy is to bound the probability of the event $E\left(\gamma_{1}, \ldots, \gamma_{4}\right)$ by the product of probabilities of crossing events in such annuli. We should be more careful though, since we have to take into account that we consider paths in $S$. For $k<4$ and for each nonempty annulus $A n n_{i}(k)$, we define $a_{i}(k)$ as the first point on $\gamma_{i-1}$ (seen as an oriented path from $a_{i-1}$ to $a_{i}$ ) that belongs to $A n n_{i}(k)$, and $b_{i}(k)$ as the last point on $\gamma_{i+k}$ (seen as an oriented path from $a_{i+k}$ to $a_{i+k+1}$ ) that belongs to $A n n_{i}(k)$. Note that such points always exist if $A n n_{i}(k) \neq \emptyset$. We then define the set $S_{i}(k)$ as the subset of $S$ with boundary that consists of four pieces (in clockwise order): the piece of $\partial B\left(x_{i}(k), 2^{\widetilde{l}_{i}(k)-3}\right)$ between $a_{i}(k)$ and $b_{i}(k)$, the piece of $\gamma_{i+k}$ from $b_{i}(k)$ to the last intersection of $\gamma_{i+k}$ with $\partial B\left(x_{i}(k), 2^{l_{i}(k)}\right)$, the piece of $\partial B\left(x_{i}(k), 2^{l_{i}(k)}\right)$ in $S$, and the piece of $\gamma_{i-1}$ from $a_{i}(k)$ to the last intersection with $\partial B\left(x_{i}(k), 2^{l_{i}(k)}\right)$. Let $L_{i}(k)$ be the common piece of the boundary of $S_{i}(k)$ and $\partial B\left(x_{i}(k), 2^{\widetilde{l}_{i}(k)-3}\right)$ between $a_{i}(k)$ and $b_{i}(k)$ (Fig. 6).

For $k<4$ and for each nonempty annulus $A n n_{i}(k)$, let $E_{i}(k)$ be the event that there exist $k$ disjoint paths from $B\left(x_{i}(k), 2^{l_{i}(k)}\right)$ to $L_{i}(k)$ in $S_{i}(k)$ such that the order and the status of paths (occupied or vacant) are induced by the order and the status of $P_{i}, \ldots, P_{i+k-1}$. For $k<4$ and for each empty annulus $A n n_{i}(k)$, let $E_{i}(k)$ be the sure event. Let $E_{i}(4)$ be the event that the annulus $A n n_{i}(4)$ is crossed by two open and two closed dual paths such that the open paths are separated by the closed paths. 


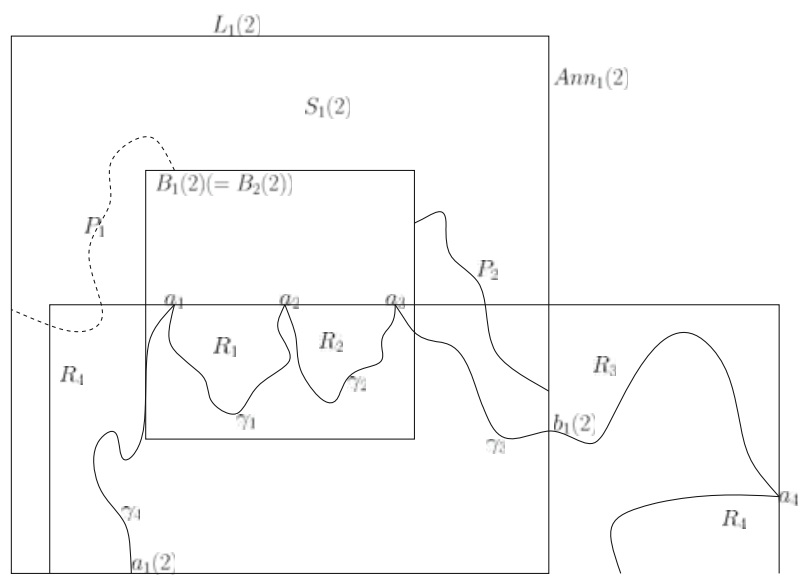

Fig. 6 The small box is $B\left(x_{1}(2), 2^{l_{1}(2)}\right)$ and the big box is $\partial B\left(x_{1}(2), 2^{\widetilde{l}_{1}(2)-3}\right)$. The event $E_{1}(2)$ occurs if there is a closed dual path $P_{1}$ and an open path $P_{2}$ from $B\left(x_{1}(2), 2^{l_{1}(2)}\right)$ to $L_{1}(2)$ in $S_{1}(2)$

Since the sets $S_{i}(k)$ and $A n n_{i}(4)$ are disjoint, we obtain

$$
\mathbb{P}\left(E\left(\gamma_{1}, \ldots, \gamma_{4}\right)\right) \leq \prod_{i, k=1}^{4} \mathbb{P}\left(E_{i}(k)\right) .
$$

It remains to prove an arm separation statement (analogous to Lemmas 4 and 5 in [13]) for each of the crossing events $E_{i}(k)$. Once this is done, we can proceed similarly to the proof of (2.43) in [13]. We can 'glue' those crossing into solid paths from the $\gamma_{i}$ 's to $\partial B\left(2^{n}\right)$ in such a way that the event $\widetilde{E}\left(\gamma_{1}, \ldots, \gamma_{4}\right)$ occurs, and there exists a constant $C_{5}$ such that for any choice of $\gamma_{i}$ 's, $\prod_{i, k=1}^{4} \mathbb{P}\left(E_{i}(k)\right) \leq C_{5} \mathbb{P}\left(\widetilde{E}\left(\gamma_{1}, \ldots, \gamma_{4}\right)\right)$. Since this is a standard application of the RSW theorem (Section 11.7 in [9]) and the generalized FKG inequality [13, Lemma 3], we omit the details.

The main difficulty in the proof of the arm separation statement for $E_{i}(k)$ is that the boundary of $S_{i}(k)$ is irregular. In the proof of Lemma 4 in [13] it was enough to make sure that with high probability (uniformly in $r$ ) all the crossings of $\operatorname{Ann}\left(2^{r}, 2^{r+1}\right)$ have well-separated extremities. In our case, it is not enough to know that with high probability (uniformly in $r$ ) all the crossings of $A n n\left(x_{i}(k) ; 2^{r}, 2^{r+1}\right.$ ) in $S_{i}(k)$ are well-separated. It is possible that their extremities are "trapped" in the sense that they cannot be connected or can only be connected through narrow bottlenecks to $L_{i}(k)$ in $S_{i}(k) \backslash B\left(x_{i}(k), 2^{r+1}\right)$. An application of the RSW theorem shows that this is very unlikely. The remaining strategy of the proof is similar to the proof of Lemma 4 in [13], and we omit it. The interested reader may find the details in the second version of [5].

\section{Proof of Theorem 1.9}

We prove only the first statement; the proof of the second is similar (see the proof of the second statement in [10, Theorem 3]). We follow the same method used in 
[10, Theorem 3] but because many difficulties arise, we present details of the entire proof. Pick an edge $e$ and let $n=2|e| / 3$ (this choice makes $e$ in the middle of $\operatorname{Ann}(n, 2 n))$. Let $\epsilon>0$.

Step 1. First we give a lower bound for the probability that $e \in \mathcal{O}$. The constant $C_{*}$ will be determined later. Consider the event $D_{e}$ that

1. there exist $p_{c}$-open circuits around the origin in the annuli $A n n(n / 2, n)$ and $\operatorname{Ann}(2 n, 4 n)$;

2. there exist two disjoint $p_{c}$-open paths, one connecting $e_{y}$ to the circuit in $\operatorname{Ann}(2 n, 4 n)$ and one connecting $e_{x}$ to the circuit in $\operatorname{Ann}(n / 2, n)$;

3. there exists a $\left(2 p_{n}-p_{c}\right)$-closed dual circuit with one defect around 0 in the annulus $\operatorname{Ann}(n, 2 n)^{*}$ which includes the edge $e^{*}$ as its defect;

4. $\tau_{e} \in\left[p_{n}, 2 p_{n}-p_{c}\right)$; and

5. the $p_{c}$-open circuit in $\operatorname{Ann}(2 n, 4 n)$ is connected to $\infty$ by a $p_{n}$-open path.

By RSW arguments, [4, Lemma 6.3] and the fact that $L\left(2 p_{n}-p_{c}\right)$ is comparable with $n$ (see e.g. [13, (4.35)]),

$$
\mathbb{P}\left(D_{e}\right) \asymp\left(p_{n}-p_{c}\right) \mathbb{P}_{c r}\left(A_{n}^{2,2}\right),
$$

where $A_{n}^{2,2}$ is the event that the edge $e-e_{x}$ (we recall this notation means $\left\langle 0, e_{y}-e_{x}\right\rangle$ ) is connected to $\partial B(n)$ by two disjoint $p_{c}$-open paths and $\left(e-e_{x}\right)^{*}$ is connected to $\partial B(n)^{*}$ by two disjoint $p_{c}$-closed dual paths such that the open and closed paths alternate. Since $D_{e}$ implies that $e \in \mathcal{O}$, we have for all $e$,

$$
\mathbb{P}(e \in \mathcal{O}) \geq C_{1}\left(p_{n}-p_{c}\right) \mathbb{P}_{c r}\left(A_{n}^{2,2}\right)
$$

Step 2. Let $A_{N, M}\left(e_{x}, p_{c}\right)$ be the event that there is a $p_{c}$-open circuit with 2 defects around $e_{x}$ in $\operatorname{Ann}\left(e_{x}, N, M\right)$. We will show that $\mathbb{P}\left(A_{N, M}\left(e_{x}, p_{c}\right), \theta_{e} E \mid e \in \mathcal{O}\right)$ is close to $\mathbb{P}\left(\theta_{e} E \mid e \in \mathcal{O}\right)$ for certain values of $N<M$. To this end, recall the definition of the event $H_{n, k}$ in (5.2) and write $H$ for the event $H_{n, 1}$. By (5.3) and (7.2), we can choose $C_{*}$ independent of $n$ such that

$$
\mathbb{P}\left(\theta_{e} E, H^{c} \mid e \in \mathcal{O}\right)<\epsilon .
$$

When the event $H$ occurs, the invasion enters the $p_{n}(1)$-open infinite cluster before it reaches $e$. Hence if $e$ is an outlet, then $e$ must be connected to $\partial B\left(e_{x}, n / 4\right)$ by two disjoint $p_{n}(1)$-open paths and $e^{*}$ must be connected to $\partial B\left(e_{x}, n / 4\right)^{*}$ by two disjoint $p_{c}$-closed dual paths such that the open and closed paths alternate and are all disjoint. Also, the weight $\tau_{e}$ must be in the interval $\left[p_{c}, p_{n}(1)\right]$. If, in addition, the event $A_{N, M}\left(e_{x}, p_{c}\right)$ does not occur, then there must be yet another $p_{c}$-closed dual path from $B\left(e_{x}, N\right)^{*}$ to $\partial B\left(e_{x}, M\right)^{*}$. This crossing has the property that it is disjoint from the two $p_{c}$-closed paths which are already present; however, it does not need to be disjoint from the $p_{n}(1)$-open crossings. Therefore, $\mathbb{P}\left(\theta_{e} E, H, A_{N, M}\left(e_{x}, p_{c}\right)^{c}, e \in \mathcal{O}\right)$ is at most

$$
\leq C_{2}\left(p_{n}(1)-p_{c}\right) \mathbb{P}\left(A_{n}^{2,2}\left(p_{n}(1), p_{c}\right)\right) \mathbb{P}\left(A_{N, M}^{2,3 *}\left(p_{n}(1), p_{c}\right) \mid A_{N, M}^{2,2}\left(p_{n}(1), p_{c}\right)\right),
$$


where $A_{N, M}^{2,2}(p, q)$ denotes the event that $B(N)$ is connected to $\partial B(M)$ by two $p$-open paths and that $B(N)^{*}$ is connected to $\partial B(M)^{*}$ by two $q$-closed paths so that the open and closed paths alternate and are all disjoint. The symbol $A_{N, M}^{2,3 *}(p, q)$ signifies the event that $A_{N, M}^{2,2}(p, q)$ occurs but that there is an additional $q$-closed path connecting $B(N)$ to $\partial B(M)$ which is disjoint from the two other $q$-closed paths but not necessarily from the two $p$-open paths. The above inequality, along with the estimate (7.2), gives that $\mathbb{P}\left(\theta_{e} E, H, A_{N, M}\left(e_{x}, p_{c}\right)^{c} \mid e \in \mathcal{O}\right)$ is at most

$$
\frac{C_{2}\left(p_{n}(1)-p_{c}\right) \mathbb{P}\left(A_{n}^{2,2}\left(p_{n}(1), p_{c}\right)\right)}{C_{1}\left(p_{n}-p_{c}\right) \mathbb{P}_{c r}\left(A_{n}^{2,2}\right)} \mathbb{P}\left(A_{N, M}^{2,3 *}\left(p_{n}(1), p_{c}\right) \mid A_{N, M}^{2,2}\left(p_{n}(1), p_{c}\right)\right) .
$$

From (2.9) and Lemma 6.3 in [4], we can deduce

$$
\frac{C_{2}\left(p_{n}(1)-p_{c}\right) \mathbb{P}\left(A_{n}^{2,2}\left(p_{n}(1), p_{c}\right)\right)}{C_{1}\left(p_{n}-p_{c}\right) \mathbb{P}_{c r}\left(A_{n}^{2,2}\right)} \leq C_{3}\left(C_{*} \log n\right)^{2},
$$

so that

$$
\begin{aligned}
& \mathbb{P}\left(\theta_{e} E, H, A_{N, M}\left(e_{x}, p_{c}\right)^{c} \mid e \in \mathcal{O}\right) \\
& \quad \leq C_{3}\left(C_{*} \log n\right)^{2} \mathbb{P}\left(A_{N, M}^{2,3 *}\left(p_{n}(1), p_{c}\right) \mid A_{N, M}^{2,2}\left(p_{n}(1), p_{c}\right)\right) .
\end{aligned}
$$

The above can be made less than $\epsilon$ provided that $M / N$ grows fast enough with $n$. Let us assume this for the moment; we shall choose precise values for $M$ and $N$ at the end of the proof. Therefore, using (7.3), we have

$$
\left|\mathbb{P}\left(\theta_{e} E \mid e \in \mathcal{O}\right)-\mathbb{P}\left(\theta_{e} E, H, A_{N, M}\left(e_{x}, p_{c}\right) \mid e \in \mathcal{O}\right)\right|<2 \epsilon
$$

Step 3. We now condition on the outermost $p_{c}$-open circuit with 2 defects in $\operatorname{Ann}\left(e_{x}, N, M\right)$. For any circuit $\mathcal{C}$ with 2 defects around the origin in the annulus $\operatorname{Ann}(N, M)$, let $D(\mathcal{C})$ be the event that it is the outermost $p_{c}$-open circuit with 2 defects. Notice that $D(\mathcal{C})$ depends only on the state of edges on or outside $\mathcal{C}$. For distinct $\mathcal{C}, \mathcal{C}^{\prime}$ (i.e. the sets of edges in $\mathcal{C}$ and $\mathcal{C}^{\prime}$ are different or the sets of edges in $\mathcal{C}$ and $\mathcal{C}^{\prime}$ are the same but the defects are different), the events $D(\mathcal{C}), D\left(\mathcal{C}^{\prime}\right)$ are disjoint. Therefore, the second term of (7.6) is equal to

$$
\frac{1}{\mathbb{P}(e \in \mathcal{O})} \sum_{\mathcal{C} \subset \operatorname{Ann}(N, M)} \mathbb{P}\left(\theta_{e} E, H, \theta_{e} D(\mathcal{C}), e \in \mathcal{O}\right),
$$

where it is implied that in the sum, and in future sums like it, we only use circuits which enclose the origin.

Step 4. Let $Q\left(\theta_{e} \mathcal{C}\right)$ be the event that there exists $f \neq e$ interior to $\theta_{e} \mathcal{C}$ with $\tau_{f} \in\left[p_{c}, p_{n}(1)\right]$. We will now show that with high probability, the event $Q\left(\theta_{e} \mathcal{C}\right)$ does not occur. In other words, we will bound the probability of the event $\left\{H, Q\left(\theta_{e} \mathcal{C}\right)\right.$, $\left.\theta_{e} D(\mathcal{C}), e \in \mathcal{O}\right\}$. Supposing that this event occurs, then both $\tau_{e} \in\left[p_{c}, p_{n}(1)\right)$ and 
$A_{M, n}^{2,2}\left(p_{n}(1), p_{c}\right)$ must occur. Notice that the events $A_{M, n}^{2,2}\left(p_{n}(1), p_{c}\right), \theta_{e} D(\mathcal{C}),\left\{\tau_{e} \in\right.$ $\left.\left[p_{c}, p_{n}(1)\right)\right\}$, and $Q\left(\theta_{e} \mathcal{C}\right)$ are all independent. Hence $\mathbb{P}\left(H, Q\left(\theta_{e} \mathcal{C}\right), \theta_{e} D(\mathcal{C}), e \in \mathcal{O}\right)$ is at most

$$
\begin{aligned}
& \mathbb{P}\left(A_{M, n}^{2,2}\left(p_{n}(1), p_{c}\right)\right) \mathbb{P}\left(\theta_{e} D(\mathcal{C})\right) \mathbb{P}\left(Q\left(\theta_{e} \mathcal{C}\right)\right) \mathbb{P}\left(\tau_{e} \in\left[p_{c}, p_{n}(1)\right)\right) \\
& \quad \leq C_{4} M^{2} \frac{\left(p_{n}(1)-p_{c}\right)^{2}}{\mathbb{P}\left(A_{M}^{2,2}\left(p_{n}(1), p_{c}\right)\right)} \mathbb{P}\left(A_{n}^{2,2}\left(p_{n}(1), p_{c}\right)\right) \mathbb{P}\left(\theta_{e} D(\mathcal{C})\right)
\end{aligned}
$$

where in the last inequality we used Corollary 6.1 from [4]. Consequently,

$$
\begin{aligned}
\mathbb{P}\left(H, Q\left(\theta_{e} \mathcal{C}\right), \theta_{e} D(\mathcal{C}) \mid e \in \mathcal{O}\right) \leq & {\left[\frac{C_{4} M^{2}\left(p_{n}(1)-p_{c}\right)}{\mathbb{P}_{p_{n}(1)}\left(A_{M}^{2,2}\right)}\right] } \\
& \times\left[\frac{\left(p_{n}(1)-p_{c}\right) \mathbb{P}\left(A_{n}^{2,2}\left(p_{n}(1), p_{c}\right)\right)}{C_{1}\left(p_{n}-p_{c}\right) \mathbb{P}_{c r}\left(A_{n}^{2,2}\right)}\right] \mathbb{P}\left(\theta_{e} D(\mathcal{C})\right),
\end{aligned}
$$

which, by (7.4), is at most

$$
\frac{C_{5}\left(C_{*} \log n\right)^{2} M^{2}}{\mathbb{P}_{p_{n}(1)}\left(A_{M}^{2,2}\right)}\left(p_{n}(1)-p_{c}\right) \mathbb{P}\left(\theta_{e} D(\mathcal{C})\right) .
$$

As long as $M$ is not too big, from $\mathbb{P}_{p_{n}(1)}\left(A_{M}^{2,2}\right) \asymp \mathbb{P}_{c r}\left(A_{M}^{2,2}\right) \geq c M^{-2}$ (see, e.g., Theorem 24 and Theorem 27 in [18]), we get an upper bound of

$$
C_{6}\left(C_{*} \log n\right)^{2} M^{4}\left(p_{n}(1)-p_{c}\right) \mathbb{P}\left(\theta_{e} D(\mathcal{C})\right)<\epsilon \mathbb{P}\left(\theta_{e} D(\mathcal{C})\right) .
$$

We will be able to choose such an $M$ (in fact it will be of the order of a power of $\log n$ ), but we delay justification of this to the end of the proof. We henceforth assume that $\mathbb{P}\left(\theta_{e} E \mid e \in \mathcal{O}\right)$ is within $3 \epsilon$ of

$$
\frac{1}{\mathbb{P}(e \in \mathcal{O})} \sum_{\mathcal{C} \subset \operatorname{Ann}(N, M)} \mathbb{P}\left(\theta_{e} E, H, \theta_{e} D(\mathcal{C}), Q\left(\theta_{e} \mathcal{C}\right)^{c}, e \in \mathcal{O}\right)
$$

Step 5. We write our configuration $\omega$ as $\eta \oplus \xi$, where $\eta$ is the configuration outside or on $\theta_{e} \mathcal{C}$ and $\xi$ is the configuration inside $\theta_{e} \mathcal{C}$. We condition on both $\eta$ and $\tau_{e}$ : the summand of the numerator in (7.9) becomes

$$
\mathbb{E}\left[\mathbb{P}\left(\theta_{e} E, H, \theta_{e} D(\mathcal{C}), Q\left(\theta_{e} \mathcal{C}\right)^{c}, e \in \mathcal{O} \mid \tau_{e}, \eta\right)\right]
$$

Call the defected dual edges in $\theta_{e} \mathcal{C} e_{1}^{*}$ and $e_{2}^{*}$. Given the value of $\tau_{e}$, on the event $\theta_{e} D(\mathcal{C}) \cap H \cap Q\left(\theta_{e} \mathcal{C}\right)^{c}$, the event $\{e \in \mathcal{O}\}$ occurs if and only if all of the following occur: 


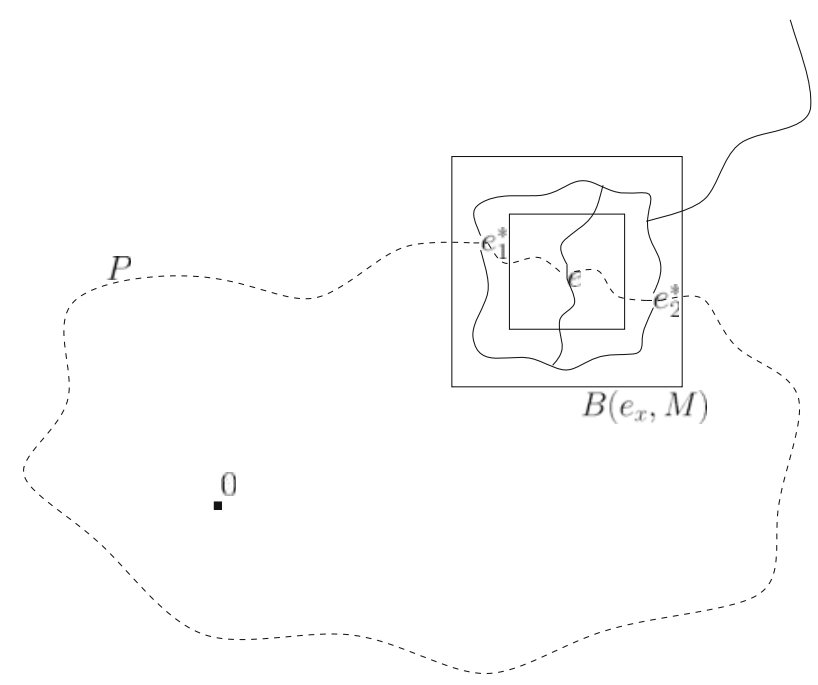

Fig. 7 The edge $e$ is connected to the circuit $\theta_{e} \mathcal{C}$ by two $\tau_{e}$-open paths (the solid lines) and two $p_{c}$-closed paths (the dotted lines). The outer dotted circuit represents the $\tau_{e}$-closed path $P$ and the circuit $\theta_{e} \mathcal{C}$ is connected to $\infty$ by a $\tau_{e}$-open path. It is assumed that the invasion from the origin touches $\theta_{e} \mathcal{C}$ before it touches $P$

1. $e$ is connected to $\theta_{e} \mathcal{C} \backslash\left\{e_{1}^{*}, e_{2}^{*}\right\}$ in the interior of $\theta_{e} \mathcal{C}$ by two disjoint $p_{c}$-open paths;

2. $e^{*}$ is connected to $\left\{e_{1}^{*}, e_{2}^{*}\right\}$ in the interior of $\theta_{e} \mathcal{C}$ by two disjoint $p_{c}$-closed dual paths so that the $p_{c}$-closed paths and the $p_{c}$-open paths from item 1 alternate and are disjoint;

3. outside of $\theta_{e} \mathcal{C}, \theta_{e} \mathcal{C}$ is connected by a $\tau_{e}$-open path to $\infty$;

4. $\tau_{e} \in\left[p_{c}, p_{n}(1)\right)$; and

5. there exists a $\tau_{e}$-closed dual path $P$ outside of $\theta_{e} \mathcal{C}$, connecting $e_{1}^{*}$ to $e_{2}^{*}$ (both of which are $\tau_{e}$-closed) such that (a) $P \cup B\left(e_{x}, M\right)^{*}$ contains a circuit around the origin and (b) the invasion graph contains a vertex from $\mathcal{C}$ before it contains an edge $f$ with $f^{*}$ from $P$.

We will denote by $e \leftrightarrow 2,2, p_{c} \theta_{e} \mathcal{C}$ the event that the first two events occur, we will denote by $\theta_{e} \mathcal{C} \leftrightarrow \tau_{e} \infty$ the third event, and we will use the symbol $X(\mathcal{C})$ for the fifth event. See Fig. 7 for an illustration of the intersection of these events. The term (7.10) becomes

$$
\begin{aligned}
& \mathbb{E}\left[\mathbb { P } \left(\theta_{e} E, H, \theta_{e} D(\mathcal{C}), e \leftrightarrow_{2,2, p_{c}} \theta_{e} \mathcal{C}, \theta_{e} \mathcal{C} \leftrightarrow \tau_{e} \infty, Q\left(\theta_{e} \mathcal{C}\right)^{c},\right.\right. \\
& \left.\left.\quad \tau_{e} \in\left[p_{c}, p_{n}(1)\right), X(\mathcal{C}) \mid \tau_{e}, \eta\right)\right] .
\end{aligned}
$$

On the event $\left\{e \leftrightarrow 2,2, p_{c} \theta_{e} \mathcal{C}\right\} \cap\left\{\theta_{e} \mathcal{C} \leftrightarrow \tau_{e} \infty\right\} \cap \tau_{e} \in\left[p_{c}, p_{n}(1)\right)$, the event $H$ occurs if and only if there exists a $p_{n}(1)$-open circuit $\mathcal{C}_{0}$ enclosing the origin in $\operatorname{Ann}(n / 4, n / 2)$ and either $\mathcal{C}_{0} \stackrel{p_{n}(1)}{\longleftrightarrow} \theta_{e} \mathcal{C}$ or $\mathcal{C}_{0} \stackrel{p_{n}(1)}{\longleftrightarrow} \infty$ outside of $\theta_{e} \mathcal{C}$. Denote by $Y$ the event that such a circuit $\mathcal{C}_{0}$ exists and that either one of the above occur. Note that $Y$ is measurable with respect to $\eta$. The term (7.11) becomes 


$$
\begin{aligned}
\mathbb{E} & {\left[\mathbb{P}\left(\theta_{e} E, Y, \theta_{e} D(\mathcal{C}), e \leftrightarrow 2,2, p_{c} \theta_{e} \mathcal{C}, \theta_{e} \mathcal{C} \leftrightarrow \tau_{e} \infty, Q\left(\theta_{e} \mathcal{C}\right)^{c}, \tau_{e} \in\left[p_{c}, p_{n}(1)\right), X(\mathcal{C}) \mid \tau_{e}, \eta\right)\right] } \\
& =\mathbb{E}\left[1_{Y} 1_{\theta_{e} D(\mathcal{C})} 1_{\theta_{e} \mathcal{C} \leftrightarrow \tau_{e}} \infty 1_{\tau_{e} \in\left[p_{c}, p_{n}(1)\right)} 1_{X(\mathcal{C})} \mathbb{P}\left(\theta_{e} E, e \leftrightarrow_{2,2, p_{c}} \theta_{e} \mathcal{C}, Q\left(\theta_{e} \mathcal{C}\right)^{c} \mid \tau_{e}, \eta\right)\right] .
\end{aligned}
$$

We now inspect the inner conditional probability. Clearly we have

$$
\begin{aligned}
\mathbb{P}\left(\theta_{e} E, e \leftrightarrow 2,2, p_{c} \theta_{e} \mathcal{C}, Q\left(\theta_{e} \mathcal{C}\right)^{c} \mid \tau_{e}, \eta\right) \leq & \mathbb{P}\left(\theta_{e} E, e \leftrightarrow_{2,2, p_{c}} \theta_{e} \mathcal{C} \mid \tau_{e}, \eta\right) \\
\leq & \mathbb{P}\left(\theta_{e} E, e \leftrightarrow_{2,2, p_{c}} \theta_{e} \mathcal{C}, Q\left(\theta_{e} \mathcal{C}\right)^{c} \mid \tau_{e}, \eta\right) \\
& +\mathbb{P}\left(Q\left(\theta_{e} \mathcal{C}\right)\right) .
\end{aligned}
$$

Using arguments similar to those that led to (7.9), one can show that the same choice of $M$ and $N$ that will make (7.8) hold will also make

$$
\frac{1}{\mathbb{P}(e \in \mathcal{O})} \sum_{\mathcal{C} \subset \operatorname{Ann}(N, M)} \mathbb{E}\left[1_{Y} 1_{\theta_{e} D(\mathcal{C})} 1_{\theta_{e} \mathcal{C} \leftrightarrow \tau_{e} \infty} 1_{\tau_{e} \in\left[p_{c}, p_{n}(1)\right)} 1_{X(\mathcal{C})} \mathbb{P}\left(Q\left(\theta_{e} \mathcal{C}\right)\right)\right]<\epsilon
$$

Therefore we conclude from (7.13) that $\mathbb{P}\left(\theta_{e} E \mid e \in \mathcal{O}\right)$ is within $4 \epsilon$ of

$$
\begin{gathered}
\frac{1}{\mathbb{P}(e \in \mathcal{O})} \sum_{\mathcal{C} \subset \operatorname{Ann}(N, M)} \mathbb{E}\left[1_{Y} 1_{\theta_{e} D(\mathcal{C})} 1_{\theta_{e} \mathcal{C} \leftrightarrow \tau_{e} \infty} 1_{\tau_{e} \in\left[p_{c}, p_{n}(1)\right)} 1_{X(\mathcal{C})}\right. \\
\left.\times \mathbb{P}\left(\theta_{e} E, e \leftrightarrow 2,2, p_{c} \theta_{e} \mathcal{C} \mid \tau_{e}, \eta\right)\right] .
\end{gathered}
$$

Step 6. Notice that since the events $\theta_{e} E$ and $e \leftrightarrow_{2,2, p_{c}} \theta_{e} \mathcal{C}$ do not depend on $\tau_{e}$ or on $\eta$, we have

$$
\mathbb{P}\left(\theta_{e} E, e \leftrightarrow_{2,2, p_{c}} \theta_{e} \mathcal{C} \mid \tau_{e}, \eta\right)=\mathbb{P}_{c r}\left(E, 0 \leftrightarrow_{2,2} \mathcal{C}\right) \text { a.s. }
$$

where $0 \leftrightarrow 2,2 \mathcal{C}$ denotes the event that the edge $e-e_{x}$ is connected to $\mathcal{C}$ by two open paths and the dual edge $\left(e-e_{x}\right)^{*}$ is connected to $\left\{\left(e_{1}-e_{x}\right)^{*},\left(e_{2}-e_{x}\right)^{*}\right\}$ by two closed paths such that all of these connections occur inside $\mathcal{C}$ and the open and closed paths alternate. The quantity $\mathbb{P}_{c r}\left(E \mid 0 \leftrightarrow_{2,2} \mathcal{C}\right)$ from the right side of (7.15) approaches $\tilde{v}^{2,2}(E)$ as long as $N \rightarrow \infty$ as $|e| \rightarrow \infty$ (this is a slight extension of Theorem 1.6) so, assuming this growth on $N$, we have

$$
\frac{1}{(1+\epsilon)} \mathbb{P}_{c r}\left(E, 0 \leftrightarrow_{2,2} \mathcal{C}\right) \leq \tilde{v}^{2,2}(E) \mathbb{P}_{c r}\left(0 \leftrightarrow_{2,2} \mathcal{C}\right) \leq \frac{1}{(1-\epsilon)} \mathbb{P}_{c r}\left(E, 0 \leftrightarrow_{2,2} \mathcal{C}\right)
$$

It is straightforward now (following the end of the proof of [10, Theorem 3]) to show that

$$
(1-\epsilon)(1-4 \epsilon) \tilde{v}^{2,2}(E)<R<(1+\epsilon)(1+4 \epsilon) \tilde{v}^{2,2}(E)
$$

where $R$ is the term which comprises the entire line of (7.15). Since $R$ is within $4 \epsilon$ of $\mathbb{P}\left(\theta_{e} E \mid e \in \mathcal{O}\right)$, all that remains is to choose $M$ and $N$ correctly. 
Step 7. Choice of $M$ and $N$. Recall, from (7.5), that we need the inequality

$$
C_{8}\left(C_{*} \log n\right)^{2} \mathbb{P}\left(A_{N, M}^{2,3 *}\left(p_{n}(1), p_{c}\right) \mid A_{N, M}^{2,2}\left(p_{n}(1), p_{c}\right)\right)<\epsilon
$$

to hold. In addition, we need to satisfy (7.8). Using the facts that $\mathbb{P}_{c r}\left(A_{M}^{2,2}\right) \geq C_{9} / M^{2}$ and

$$
\mathbb{P}\left(A_{N, M}^{2,3 *}\left(p_{n}(1), p_{c}\right) \mid A_{N, M}^{2,2}\left(p_{n}(1), p_{c}\right)\right)<C_{10}\left(\frac{N}{M}\right)^{\beta}
$$

for some $\beta>0$ (which is easily proved for what will be our choice of $M$ and $N$, and which we assume for the moment), the reader may check that a choice of

$$
N=\log n, \quad M=(\log n)^{2+2 / \beta}
$$

satisfies these two conditions for $n$ large. The reason that this choice satisfies (7.8) is that $(\log n)^{\gamma}\left(p_{n}(1)-p_{c}\right) \rightarrow 0$ for any $\gamma$ [use (2.9)] and the fact that the 4-arm exponent is strictly smaller than 2 (see, e.g., Section 6.4 in [21])).

We now prove (7.17). Let $Q(M)$ be the event that there exists an edge in $B(M)$ which has weight in the interval $\left[p_{c}, p_{n}(1)\right)$. If $Q(M)$ does not occur then the event $A_{N, M}^{2,3 *}\left(p_{n}(1), p_{c}\right)$ implies the event $A_{N, M}^{2,3}\left(p_{n}(1), p_{c}\right)$ (i.e. the same event but with all five paths disjoint). Therefore, by Reimer's inequality, $\mathbb{P}\left(A_{N, M}^{2,3 *}\left(p_{n}(1), p_{c}\right)\right)$ is at most

$$
\begin{aligned}
\mathbb{P}\left(A_{N, M}^{2,3}\left(p_{n}(1), p_{c}\right)\right)+\mathbb{P}(Q(M)) \leq & \mathbb{P}\left(A_{N, M}^{2,2}\left(p_{n}(1), p_{c}\right)\right) \mathbb{P}_{c r}\left(A_{N, M}^{0,1}\right) \\
& +|B(M)|\left(p_{n}(1)-p_{c}\right),
\end{aligned}
$$

where $A_{N, M}^{0,1}$ is the event that $B(N)$ is connected to $\partial B(M)$ by a $p_{c}$-closed path. Putting this estimate into (7.17), the term on its left is at most

$$
\mathbb{P}_{c r}\left(A_{N, M}^{0,1}\right)+|B(M)| \frac{p_{n}(1)-p_{c}}{\mathbb{P}\left(A_{N, M}^{2,2}\left(p_{n}(1), p_{c}\right)\right)} .
$$

Using the fact that

$$
\mathbb{P}\left(A_{N, M}^{2,2}\left(p_{n}(1), p_{c}\right)\right) \geq \frac{\mathbb{P}\left(A_{N, M}^{2,3}\left(p_{n}(1), p_{c}\right)\right)}{\mathbb{P}_{c r}\left(A_{N, M}^{0,1}\right)} \geq \frac{C_{11} N^{2}}{M^{2} \mathbb{P}_{c r}\left(A_{N, M}^{0,1}\right)},
$$

we see that the term (7.18) is at most

$$
\mathbb{P}_{c r}\left(A_{N, M}^{0,1}\right)\left[1+\frac{C_{12} M^{4}}{N^{2}}\left(p_{n}(1)-p_{c}\right)\right] \leq 2 \mathbb{P}_{c r}\left(A_{N, M}^{1,0}\right) \leq C_{13}\left(\frac{N}{M}\right)^{\beta}
$$


for some $\beta>0$, as we have chosen $N$ and $M$ on the order of $\log n$. This shows (7.17) and completes the proof.

Acknowledgments We would like to thank C. Newman for suggesting some of these problems. We thank R. van den Berg and C. Newman for helpful discussions. We also thank G. Pete for discussions related to arm-separation statements for multiple-armed IICs.

Open Access This article is distributed under the terms of the Creative Commons Attribution Noncommercial License which permits any noncommercial use, distribution, and reproduction in any medium, provided the original author(s) and source are credited.

\section{References}

1. Chandler, R., Koplick, J., Lerman, K., Willemsen, J.F.: Capillary displacement and percolation in porous media. J. Fluid Mech. 119, 249-267 (1982)

2. Chayes, J.T., Chayes, L., Frölich, J.: The low-temperature behavior of disordered magnets. Commun. Math. Phys. 100, 399-437 (1985)

3. Chayes, J.T., Chayes, L., Newman, C.: The stochastic geometry of invasion percolation. Commun. Math. Phys. 101, 383-407 (1985)

4. Damron, M., Sapozhnikov, A., Vágvölgyi, B.: Relations between invasion percolation and critical percolation in two dimensions. Ann. Probab. 37, 2297-2331 (2009)

5. Damron, M., Sapozhnikov, A.: arXiv:0903.4496 (2009)

6. Diestel, R.: Graph Theory, 2nd edn. Springer, New York (2000)

7. Garban, C., Pete, G.: Personal communication (2009)

8. Goodman, J.: Exponential growth of ponds for invasion percolation on regular trees (2009, preprint)

9. Grimmett, G.: Percolation, 2nd edn. Springer, Berlin (1999)

10. Járai, A.A.: Invasion percolation and the incipient infinite cluster in $2 D$. Commun. Math. Phys. 236, 311-334 (2003)

11. Kesten, H.: A scaling relation at criticality for $2 D$-percolation. Percolation theory and ergodic theory of infinite particle systems (Minneapolis, Minn., 1984-1985), IMA Vol. Math. Appl., vol. 8, pp. 203-212. Springer, New York (1987)

12. Kesten, H.: The incipient infinite cluster in two-dimesional percolation. Probab. Theory Rel. Fields 73, 369-394 (1986)

13. Kesten, H.: Scaling relations for $2 D$ percolation. Commun. Math. Phys. 109, 109-156 (1987)

14. Lenormand, R., Bories, S.: Description d'un mecanisme de connexion de liaision destine a l'etude du drainage avec piegeage en milieu poreux. C. R. Acad. Sci. 291, 279-282 (1980)

15. Nagaev, S.V.: Large deviations of sums of independent random variables. Ann. Probab. 7, 745789 (1979)

16. Newman, C., Stein, D.L.: Broken ergodicity and the geometry of rugged landscapes. Phys. Rev. E. 51, 5228-5238 (1995)

17. Nguyen, B.G.: Correlation lengths for percolation processes. Ph. D. Thesis, University of California, Los Angeles (1985)

18. Nolin, P.: Near critical percolation in two-dimensions. Electron. J. Probab. 13, 1562-1623 (2008)

19. Reimer, D.: Proof of the van den Berg-Kesten conjecture. Combin. Probab. Comput. 9, 27-32 (2000)

20. van den Berg, J., Járai, A.A., Vágvölgyi, B.: The size of a pond in $2 D$ invasion percolation. Electron. Comm. Probab. 12, 411-420 (2007)

21. Werner, W.: Lectures on two-dimensional critical percolation. arXiv: 0710.0856 (2007) 